\title{
A New Distance to M33 Using Blue Supergiants and the FGLR Method
}

\author{
Vivian U, Miguel A. Urbaneja, Rolf-Peter Kudritzki, Bradley A. Jacobs, Fabio Bresolin \\ Institute for Astronomy, University of Hawaii, 2680 Woodlawn Drive, Honolulu, HI 96822 \\ vivian@ifa.hawaii.edu, urbaneja@ifa.hawaii.edu, kud@ifa.hawaii.edu, \\ bjacobs@ifa.hawaii.edu, bresolin@ifa.hawaii.edu \\ and \\ Norbert Przybilla \\ Dr. Remeis-Sternwarte Bamberg, Sternwartstrasse 7, D-96049 Bamberg, Germany \\ przybilla@sternwarte.uni-erlangen.de
}

\begin{abstract}
The quantitative spectral analysis of medium resolution optical spectra of A and B supergiants obtained with DEIMOS and ESI at the Keck Telescopes is used to determine a distance modulus of $24.93 \pm 0.11 \mathrm{mag}$ for the Triangulum Galaxy M33. The analysis yields stellar effective temperatures, gravities, interstellar reddening, and extinction, the combination of which provides a distance estimate via the Flux-weighted Gravity-Luminosity Relationship (FGLR). This result is based on an FGLR calibration that is continually being polished. An average reddening of $<E(B-V)>\sim 0.08 \mathrm{mag}$ is found, with a large variation ranging from 0.01 to $0.16 \mathrm{mag}$ however, demonstrating the importance of accurate individual reddening measurements for stellar distance indicators in galaxies with evident signatures of interstellar absorption. The large distance modulus found is in good agreement with recent work on eclipsing binaries, planetary nebulae, long period variables, RR Lyrae stars, and also with HST observations of Cepheids, if reasonable reddening assumptions are made for the Cepheids. Since distances based on the tip of the red giant branch (TRGB) method found in the literature give conflicting results, we have used HST ACS $V$ - and $I$-band images of outer regions of M 33 to determine a TRGB distance of $24.84 \pm 0.10$ mag, in basic agreement with the FGLR result. We have also determined stellar metallicities and discussed the metallicity gradient in the disk of M33. We find metallicity of $Z_{\odot}$ at the center and $0.3 Z_{\odot}$ in the outskirts at a distance of one isophotal radius.
\end{abstract}


The average logarithmic metallicity gradient is $-0.07 \pm 0.01 \mathrm{dex} \mathrm{kpc}^{-1}$. However, there is a large scatter around this average value, very similar to what has been found for the H II regions in M 33 .

Subject headings: galaxies: distances and redshifts — galaxies: individual (M33) — stars: abundances — stars: early type — supergiants

\section{Introduction}

Modern astronomy has advanced to a stage where cosmological parameters are claimed to be determined with hitherto unknown precision (e.g. Frieman et al. 2008). At the same time, however, we are confronted with the fact that distances to many nearby galaxies are disturbingly uncertain. An interesting and important example is the distance to the Triangulum Galaxy M33 (see Bonanos et al. 2006 for a summary). Several independent distance determination techniques have been employed for this galaxy since the beginning of this decade, including Cepheids and RR Lyrae (Freedman et al. 1991; Lee et al. 2002; Sarajedini et al. 2006), tip of the red giant branch (TRGB) and red clump stars (Rizzi et al. 2007; Galleti et al. 2004; McConnachie et al. 2004; Tiede et al. 2004; Kim et al. 2002), planetary nebulae (Ciardullo et al. 2004), horizontal branch stars (Saraiedini et al. 2000), long period variables (Pierce et al. 2000), detached eclipsing binary (Bonanos et al. 2006), and water masers (Brunthaler et al. 2005). The shocking result is that the distance moduli obtained with these different methods differ by as much as 0.6 magnitudes, which is more than 30 percent in linear distance. Even when the water maser distance were discounted in this comparison as justified by its large intrinsic uncertainty of $0.45 \mathrm{mag}$, the spread in distance moduli is still about 0.4 magnitudes, significantly higher than the uncertainties quoted in the individual works.

A significant part of the uncertainty comes from interstellar extinction. M 33 is a mildinclination star-forming galaxy with a patchy dusty disk that exhibits inhomogeneous intrinsic extinction. This has a considerable effect for many of the aforementioned methods. In particular, distance determination using Cepheids at $V$ and $I$ bands is severely affected, as they are part of a young stellar population in a dusty star-forming environment. As pointed out by Kim et al. (2002) and Ciardullo et al. (2004), the difference between the shorter distances obtained with Cepheids and the longer distances found with other methods would disappear given smaller reddening than assumed in the Cepheid work cited above. Interestingly, the work by Bonanos et al. (2006), which uses quantitative spectroscopy of a detached eclipsing binary and, thus, comes up with an accurate determination of reddening and extinction, yields a large distance modulus of $24.92 \mathrm{mag}$, 0.3 magnitudes larger than the one 
obtained with $V$ - and $I$-band photometry of Cepheids.

The solution to resolving this discrepancy is to use a method that is either unaffected by reddening and extinction uncertainties such as K-band photometry of Cepheids (e.g. Gieren et al. 2005), or one that includes the accurate determination of reddening for each individual star gauged in the determination of the distance modulus. An example for the latter involves the quantitative spectroscopy of massive blue supergiant stars and the use of the Flux-weighted Gravity-Luminosity Relationship (FGLR; Kudritzki et al. 2003) . In this paper we will apply this method to determine the reddening, extinction, and distance to M33.

Blue supergiants of B and A spectral types are the optically brightest stars in the universe with visual magnitudes up to $M_{V} \cong-9.5 \mathrm{mag}$. Their quantitative spectral analysis based on state-of-the-art non-LTE radiative transfer models allows for precise determination of stellar parameters, metallicity, reddening, and extinction (Urbaneja et al. 2005a; Przybilla et al. 2006; Kudritzki et al. 2008). Then, with stellar temperatures $T_{\text {eff }}$ and gravities $\log g$, one can use the de-reddened apparent stellar magnitudes and the tight relationship between flux-weighted gravity $\log g_{F}=\log g-4 \log \left(T_{\text {eff }} \times 10^{-4}\right)$ and absolute bolometric magnitude $M_{\mathrm{bol}}$, to determine a distance via the FGLR.

The concept of FGLR has been introduced by Kudritzki et al. (2003). It is based on the assumption that massive stars evolve through the Hertzsprung-Russell $(\mathrm{H}-\mathrm{R})$ diagram temperature domain of $\mathrm{B}$ and $\mathrm{A}$ spectral types at constant luminosity and mass. In a detailed spectroscopic study of blue supergiants in the nearby spiral galaxy NGC 300, which is at a distance of $1.9 \mathrm{Mpc}$, Kudritzki et al. (2008, hereafter K08) verified this concept and contributed towards the calibration of the FGLR. Urbaneja et al. (2008) then applied the FGLR method and determined the distance to the metal-poor, irregular Local Group galaxy WLM, the result from which agreed well with most recent distance determinations based on K-band photometry of Cepheids and I-band photometry of the TRGB.

Motivated by the success of previous application of the FGLR as well as the prospects of this work, we undertake an independent investigation of the distance to M33 by means of a quantitative analysis of medium resolution optical spectra of blue supergiant stars.

This paper is organized as follows: In \$2 we describe the data sample used to obtain the FGLR of M33 as well as the observations undertaken at Keck Observatory. In §3 we detail the process of the quantitative spectroscopic analysis. We present the results of the spectral analysis in $\$ 4$ and discuss stellar parameters and stellar evolution, metallicity and metallity gradient, and reddening and extinction. This provides the basis for a new distance determination using the FGLR in \$5. A discussion of the new result compared with distances determined from other methods is given in $\$ 5.3$. Finally, we present our conclusions and 
future outlook in $\S 6$,

\section{Observations}

Distance determination using the FGLR method requires a sufficiently large sample (10 to 20 objects, see K08, Urbaneja et al. 2008) of supergiants with good multi-band photometry and spectra of reasonable quality that are suitable for quantitative analysis using model atmosphere and radiative transfer techniques. We pre-selected a sample of such potential targets that fulfill the initial color-magnitude criteria described in K08. Massey et al. (2006) provides an excellent source for photometry of all these objects; spectra were collected with the Keck Telescopes on Mauna Kea. In our first observing run for this project on November 1, 2003 with good seeing (0'.6), we used the DEIMOS spectrograph with a $1200 \mathrm{l} / \mathrm{mm}$ grating yielding a dispersion of $0.33 \AA / \mathrm{px}$ and a spectral resolution (FWHM) of $1.6 \AA$. The total integration time was 135 and 93 minutes for the blue and red spectra, respectively. A total number of 49 targets were observed with one DEIMOS mask. Among these, we selected 10 objects with spectral type ranging from B3 to A3 based on the requirement of a minimum signal-to-noise ratio (SNR) needed to carry out the ensuing spectral analysis.

Additional observations of 7 more A-type supergiants were carried out under good weather conditions (clear sky, typical seeing around 1".0) on October 8, 2005 and October 4, 2007 with the ESI spectrograph (Sheinis et al. 2002). The instrument was used in echellette mode, with a slit width of 0.75 , providing $\mathrm{R} \sim 9000$ (equivalent to $0.6 \AA$ FWHM at 5500 $\AA$ ). Depending on the visual magnitude of the object, one to three exposures were acquired, with the exposure time adjusted to provide a final SNR $\sim 100$ in the continuum. Unlike in the case of DEIMOS, the ESI stars were selected to cover a wide range of galactocentric distances.

To further increase the sample size for FGLR analysis and calibration, we included

additional stars from the spectroscopic study by Urbaneja et al. (2005b). In this work, 11 B supergiants of early spectral type (B0 to B2) were analyzed quantitatively using stateof-the-art non-LTE model atmospheres to investigate the M33 metallicity gradient. While these stars were well suited for the abundance study, however, care must be taken while considering their selection as targets for the FGLR distance determination method. Two of them (B133 and 1054) were found to be multiple systems with companions that would affect magnitudes and colors. Two others (110A and OB10-10) turned out to be extreme blue hypergiants, with very high luminosities, rendering the determination of their surface gravities less reliable. For object 1137, only an upper limit for the mass loss rate could be found, which means that, because of wind contamination in the Balmer lines, only an upper 
limit for the gravity could be determined. The spectra for object 0900 suffered from strong $\mathrm{H}$ II region emission and consequently-distorted profiles of the two observed Balmer lines $\mathrm{H} \gamma$ and $\mathrm{H} \delta$, again leading to a rather uncertain stellar gravity. The remaining five objects have very well determined stellar parameters and have been incorporated as targets in the FGLR distance determination technique. This brings the total number of M33 supergiants in our sample up to 22. The list of all the objects is given in Tbl. 1,

The new ESI data were reduced in the same way as described in Urbaneja et al. (2005b). On the other hand, the DEIMOS spectra were initially reduced using the DEEP2 Reduction Software (e.g. Marinoni et al. 2001), but the resulting SNR in the short wavelength range were insufficiently low because the subroutines in the pipeline optimizes in the longer wavelength regime. Therefore, the data reduction of the DEIMOS data was carefully redone step-by-step using the standard Image Reduction and Analysis Facility (IRAF) packages. As demonstrated in Fig. 1, the final extracted spectra have a clearly improved SNR in the blue region, giving better results than the DEEP2 pipeline did. The noticeably-improved SNR allows us to identify and measure the relevant spectral features more precisely. However, even after this improvement in SNR, the poor blue sensitivity of DEIMOS (as can be seen in Fig. 1) turned out to be an extra challenge during the spectral analysis.

\section{Spectral Analysis}

The analysis method to be applied to the individual targets depends on the targets' spectral types. For early B supergiants, we use the Si II/III/IV equilibrium based on metal-lineblanketed non-LTE model atmospheres and line formation calculations that include effects of stellar winds and spherical extension to constrain effective temperatures (see Urbaneja et al. $2005 \mathrm{a}, \mathrm{b}$ ). For late B and early A spectral types, the determination of effective temperature utilizes the Balmer jump or ionization equilibrium such as Mg I/II and O I/II. These models involve LTE line-blanketed model atmospheres in hydrostatic equilibrium with planeparallel geometry and very detailed non-LTE line formation calculations (Przybilla et al. 2006; Kudritzki et al. 2008). In consequence, as a first step towards quantitative spectral analysis, we determined spectral types for all 14 newly-observed objects through a qualitative comparison with a complete set of Milky Way and SMC supergiant template spectra (see Bresolin et al. 2001; Lennon 1997). Only three objects were found to exhibit early spectral type of B3 while that of the rest ranges between B8 to A2 (see Tbl. 2).

Spectra analyzed in the FGLR studies by K08 and Urbaneja et al. (2008) had a rather low resolution of $5 \AA$. While this resolution is sufficiently good for determining stellar gravities from the Balmer lines, it precludes the use of ionization equilibria for temperature 
determination in the case of late B and early A supergiants because the weak spectral lines of the neutral species disappear in the noise of the spectra. As an alternative, the Balmer jump or the strengths of the neutral helium lines were utilized instead. However, with the spectroscopic resolution in our Keck DEIMOS and ESI spectra (1.6 and 0.6 ^ respectively), we can employ the technique of ionization equilibria in this work (see Przybilla et al. 2006).

The basis for the spectral analysis of late B and A type supergiant stars is the grid of model atmospheres and line formation calculations in K08 that spans an extensive area in $\left(T_{\text {eff }}, \log g\right)$ parameter space (see Fig. 1 in K08) and covers a large range in metallicity from $0.05 Z_{\odot}$ to $2 Z_{\odot}$. We refer to K08 for a description of the computed grid (abundances, choice of microturbulence velocities, etc.). The input physics of the model atmospheres and line formation calculations are detailed in Przybilla et al. (2006). Regarding the mid B-types, we use the unified non-LTE model atmosphere/line formation code FASTWIND (Puls et al. 2005) to create a small grid of models, with the parameters properly selected to explore the expected small parameter space covered by these three objects.

The analysis proceeds in three steps. First, projected rotational velocity is determined; second, stellar effective temperature and gravity; third, stellar metallicity. If needed, steps two and three are iterated. In the following, we describe the individual steps.

\subsection{Rotational Velocities}

Projected rotational velocities $v \sin i$ of late B and early A supergiants are usually between 30 to $70 \mathrm{~km} \mathrm{~s}^{-1}$ (Howarth 2004) comparable to or somewhat larger than the resolution of the DEIMOS and ESI spectrographs, respectively. In other words, we have to determine the amount of rotational line broadening in addition to instrumental broadening for each individual star before we start the spectral analysis via comparing synthetic with observed line profiles.

With the large number of objects to be analyzed, it is important to have a simple method to accurately estimate $v \sin i$ that is independent of stellar parameters as well as the details of the final atmospheric model synthesizing the observed spectrum. A good approach is to look at lines that are weak enough to not suffer from pressure broadening to a large degree but still sufficiently strong such that the rotation-broadened profile shape can be well determined (Gray 1992). The Mg II $\lambda 4481$ line, the Si II $\lambda 6347,6371$ lines and the Fe II $\lambda 4515,4535$ lines are ideal candidates for late B through early A supergiants while the Si III lines at $\lambda 4552,4567,4574$ are useful for early B supergiants. For those lines we construct a Gaussian profile with very narrow FWHM $(0.1 \AA)$ that has the same equivalent width as 
the observed line. We then convolve this initial profile with the instrumental profile and a rotational profile, where we steadily increase $v \sin i$. An example in the case of the DEIMOS spectra is given in Fig. 2, A $\chi^{2}$ minimization of the difference between the observed and calculated profiles in turn indicates projected rotational velocities. We note a caveat that Mg II $\lambda 4481$ is a blend of two strong components separated by $0.2 \AA$; determination of $v \sin i$ with a single Gaussian may tend to overestimate $v \sin i$ in these cases.

The lower spectral resolution and SNR achieved for the DEIMOS data do not allow us to disentangle rotation and macroturbulence. Therefore, the value of $v \sin i$ thus obtained incorporates macroturbulence and might overestimate the true rotational velocity. This, however, will not affect the determination of stellar parameters and subsequent FGLR distance analysis. The situation is more optimistic for the late B and early A supergiants observed with ESI that have significantly better spectral resolution and SNR; we thus decided to apply a two-step procedure. First, $v \sin i$ was determined by a Fourier transform of individual lines (such as the ones mentioned previously) as described in Gray (1992). With this value fixed, the macroturbulence velocity was then established by a by-eye fit of the observed profile similar to that done in Przybilla et al. (2006). The early B supergiant sample from Urbaneja et al. (2005b) were in a situation similar to that of our DEIMOS spectra: in order to obtain a reasonable SNR, the ESI spectra had to be binned to the detriment of the resolution, which did not allow then to discern between rotation and macroturbulence.

A list of rotational and macroturbulence velocities for our sample of supergiants is given in Tbl. 2. The uncertainties are on the order of $5 \mathrm{kms}^{-1}$.

\subsection{Gravity and Effective Temperature}

The standard technique for determining gravities and effective temperatures of late B and early A supergiants is described in detail by Przybilla et al. (2006) and K08. We apply a very similar but somewhat more efficient technique in this work.

The first step involves using information about gravity and effective temperature that are provided by a fit of the Balmer line profiles. Balmer lines are an excellent indicator of stellar gravity because of their strong density dependence on pressure broadening through the Stark effect. An example is given in Fig. 3 for object No. 11 of our sample. At a fixed effective temperature, the step size of each gravity fit on the grid is 0.05 dex. Since the excitation of the second level of hydrogen and the ionization of hydrogen is temperature-dependent, the strength of the Balmer lines also depends on effective temperature. This means that the Balmer lines may be fitted just as well as higher (lower) effective temperature and higher 
(lower) gravity than shown for the example in Fig. 3. The corresponding fit curve on the $\left(\log g, T_{\text {eff }}\right)$-plane is demonstrated in Fig. 4. Since the major motivation for this work is a determination of the distance to M33 using the stellar flux-weighted gravity $\log g_{F}$, we also plot the same fit curve for the Balmer lines on the $\left(\log g_{F}, T_{\text {eff }}\right)$-plane. We note that for stars sufficiently hot, $\log g_{F}$ can be solely determined by fitting the Balmer lines. The physics behind this phenomenon is discussed in detail in K08.

While the analysis of the Balmer lines provides a strong constraint for gravity and temperature, we need a second, more temperature-dependent spectral feature to further constrain the effective temperature along the Balmer line fit curve in Fig. 4. The most accurate solution is to use ionization equilibria such as Mg I/II for late B and early A supergiants (see Przybilla et al. 2006) and Si II/III/IV for early B supergiants (see Urbaneja et al. 2005b). Fig. 5 demonstrates how this ionization equilibrium changes with $\left(T_{\text {eff }}, \log g\right)$ pairs and sets the best-fit effective temperature of object No. 5 to be $8750 \pm 250 \mathrm{~K}$.

Because of the relatively high resolution and high SNR $(\sim 100)$, the ionization equilibrium method works extremely well for all our targets with ESI spectra. The DEIMOS spectra exhibit lower resolution and lower SNR $(\sim 30-70)$, but the method is still successful for many of the remaining targets, as shown in Fig. 5. However, there were some DEIMOS targets for which no Mg I lines could be observed because either the stars were too hot or the spectra were too noisy, or both. For such cases we applied an alternative technique introduced by K08 that made use of the very strong temperature dependence of the neutral helium lines in the temperature range from $9000 \mathrm{~K}$ to $13000 \mathrm{~K}$. An example for such a DEIMOS case is given in Fig. 6, where a fit of the He I lines of object No. 1 yields $T_{\text {eff }}=$ $10000 \pm 500 \mathrm{~K}$. The fit of the Balmer lines for the same object is also presented in the figure. Naturally, using the He I lines requires making an assumption about the helium abundance, which is slightly enhanced in the atmospheres of late B and early A supergiants but varies only between $y=N(H e) /(N(H e)+N(H))=0.11$ to 0.13 according to the high resolution, high SNR quantitative spectroscopy by Przybilla et al. (2006) and Schiller \& Przybilla (2008). We have adopted $y=0.12$ for the fit in Fig. 6. We note, though, that the influence of temperature variation on the HeI lines in this temperature range is much stronger than, for instance, changing y from the solar value of 0.09 to 0.13 . We also note that for all cases in this temperature range, where we were able to constrain the effective temperature with ionization equilibria (e.g. all our objects observed with ESI), the agreement of the observed He I lines and computed models is excellent, lending support to the reliability of He I method.

Tbl. 2 gives the effective temperatures and gravities thus obtained along with their associated uncertainties. Note that in most cases the errors for log $g$ are larger than that for $\log g_{F}$. The reason is that the gravity fit curve has a stronger dependence on temperature 
than the flux-weighted gravity fit curve, as illustrated in the upper and lower panels of Fig. 4 , respectively (see K08 for a detailed discussion of this effect).

\subsection{Metallicity}

The use of ionization equilibrium such as Mg I/II as illustrated in Fig. 5 presumes a stellar magnesium abundance or, at least, a stellar metallicity (the dependence of the Balmer or Helium lines on metallicity is very weak and can be neglected). As a starting point for $T_{\text {eff }}$ and $\log g$ determination, we have adopted metallicities in agreement with the metallicities and the metallicity gradient found by Urbaneja et al. (2005b). After constraints have been placed on the values of $T_{\text {eff }}$ and $\log g$, we then check whether the initial metallicity assumption was correct. This process is exemplified in Fig. 7 and detailed as follows. We define spectral windows, which are dominated by metal lines and for which a good definition of the continuum is possible. We then select all available models with different metallicities (typically ranging from $[Z]=-1.3$ to $0.3 \mathrm{dex}$, with $\left.[Z]=\log Z / Z_{\odot}\right)$ at the corresponding $\left(T_{\text {eff }}\right.$, $\log g$ ) and, for each of these models, carry out a pixel-by-pixel comparison of the calculated and normalized fluxes within each spectral window. The metallicity over each spectral range is thus the metallicity associated with the minimum $\chi^{2}$-value; the average $[Z]$ from all windows then gives an estimate of the stellar metallicity. If the value of $[Z]$ thus obtained differs from that used for the ionization equilibrium by more than 0.1 dex, we reiterate the $T_{\text {eff }}$ and $\log g$ determination process using this new $[Z]$ until the metallicity measurements converge. This procedure is repeated for models at the extremes of the error box for $T_{\text {eff }}$ and $\log g$, which, together with the dispersion in $[Z]$ obtained in all spectral windows, defines the uncertainty in $[Z]$ (see K08 for details). The stellar metallicities with uncertainties for our sample of supergiants are given in Tbl. 2. Note that the metallicities obtained via this technique for the late B and early A supergiants reflect mostly the abundances of heavy elements such as iron and titanium. For the early B supergiants, the same method as was described in Urbaneja et al. (2005b) was applied: the metallicities are an average of oxygen, magnesium and silicon abundances.

\subsection{Interstellar Reddening, De-reddened Magnitudes, Stellar Luminosities, Radii, and Masses}

With effective temperature, gravity, and metallicity constrained from our spectral analysis, we know the intrinsic energy distribution of our target stars from the flux distribution

of the model atmosphere computed with the final stellar parameters (see Przybilla et al. 
2006; Kudritzki et al. 2008; Urbaneja et al. 2008). Comparing with the observed fluxes from Massey et al. (2006) and applying the interstellar extinction law found by Cardelli et al. (1989), we can obtain an accurate estimate of interstellar reddening $E(B-V)$ for each individual supergiant (see K08 for details). Assuming $A_{V}=3.1 E(B-V)$ for the relationship between reddening and visual extinction yields the de-reddened apparent bolometric magnitude $m_{\text {bol }}$ given by

$$
m_{\mathrm{bol}}=m_{V}-A_{V}+B C,
$$

where $m_{V}$ is the observed apparent visual magnitude; $B C$ is the bolometric correction, which is also given by the flux distribution of the model atmosphere calculated with the final stellar parameters. Note that K08 give an accurate analytical formula to compute $B C$ as a function of $T_{\text {eff }}, \log g$, and $[Z]$. Stellar photometry including intrinsic colors, reddening, and bolometric correction is summarized in Tbl. 3 ,

Assuming an appropriate distance modulus, for instance as determined via the FGLR method (see below), the de-reddened apparent bolometric magnitude $m_{\text {bol }}$ can subsequently be used to evaluate the absolute bolometric magnitude $M_{\text {bol }}$ or, equivalently, the stellar luminosity $\log \left(L / L_{\odot}\right)$. Stellar luminosities coupled with effective temperatures then yield stellar radii. Tbl. 4 lists the absolute bolometric magnitudes and radii obtained in this way.

As explained by K08, there are two ways to determine stellar masses. We can use stellar gravities together with the radii to directly calculate masses; masses subsequently determined are referred to as spectroscopic masses. Alternatively, masses can be estimated by comparing the location of our target stars on the H-R diagram with evolutionary

tracks (Maeder \& Mevnet 2005); these masses are called evolutionary masses. Both masses along with their uncertainties are given in Tbl. 4.

\section{Results}

In this section we discuss the main results of our quantitative spectral analysis that are compiled in Tbl. 2, Tbl. 3, and Tbl. 4.

\subsection{Interstellar Reddening and Extinction}

Fig. 8 shows the distribution of interstellar reddening $E(B-V)$ among the stars in our sample. We find a wide range from 0.01 to $0.16 \mathrm{mag}$ with an average value of $<E(B-$ $V)>=0.083 \mathrm{mag}$. The individual values are significantly lower than $E(B-V)=0.20 \mathrm{mag}$, the reddening value as adopted in the HST distance scale Key Project study of Cepheids 
by Freedman et al. (1991) (see also Lee et al. 2002). This will have important repercussions for the discussion of the distance to M33 in $\$ 5.3$. We note that our average value is higher than the foreground value of $E(B-V)=0.04 \mathrm{mag}$, which has been derived from the reddening maps by Schlegel et al. (1998). We also note that the wide range in reddening found in this study is similar to the range in NGC 300 derived by K08 and demonstrates the need for reliable individual reddening determinations.

An independent method to acquire information about interstellar reddening in M33 is the study of the Balmer decrement of H II regions. While H II regions and the associated stars within are generally younger than A and B supergiants as well as Cepheids, which in many cases have already migrated into the field, and while the average reddening of $\mathrm{H}$ II regions is hence very likely somewhat higher, it is still useful to discuss $E(B-V)$ values obtained with this technique. Most recently, Rosolowsky \& Simon (2008) in their comprehensive study of M $33 \mathrm{H}$ II regions have published reddening values $\mathrm{c}\left(\mathrm{H}_{\beta}\right)$ for a large sample. Adopting $E(B-V)=0.676 \mathrm{c}\left(\mathrm{H}_{\beta}\right)$ (Magrini et al. 2007b), we produce Fig. 9 with reddening values from Rosolowsky \& Simon (2008), where we plot $E(B-V)$ as a function of angular galactic distance, in units of $R_{25}$ (de Vaucouleurs et al. 1995). The result is very informative: there is a large scatter in reddening at all distances; however, the average $E(B-V)$ value (excluding the three most extreme cases with $E(B-V) \geq 0.6 \mathrm{mag})$ is $\langle E(B-V)\rangle=0.11 \mathrm{mag}$, which is in close agreement to what has been found for our blue supergiants. We note that Magrini et al. (2007b) quote an average reddening of $\langle E(B-V)>=0.22$ mag from their study of H II regions; however, their work systematically overestimates the reddening value, since, when measuring Balmer emission line fluxes and the Balmer decrement, they have not corrected for the effect of the underlying stellar Balmer absorption.

\subsection{Stellar Properties}

Fig. 10 (upper panel) shows the location of all targets on the (log $g, \log T_{\text {eff }}$ )-plane along with evolutionary tracks (Meynet \& Maeder 2003) calculated for solar metallicity that include effects of rotation and anisotropic mass loss. In this diagram, the data points of the stars stand independent of any assumption about the distance to the galaxy and rely completely on quantitative stellar spectroscopy. There is an indication of two evolutionary sequences. The six early B supergiants (No. 17 to 22 on our target list) and one late B supergiant (No. 16) align with the $40 M_{\odot}$ evolutionary track, while the others appear to be in the 20 to $25 M_{\odot}$ range. One object (No. 4) that coincides with the $15 M_{\odot}$ evolutionary track seems to be of lower mass. The fact that all the early B supergiants are more massive is a selection effect: the objects have been chosen according to their apparent 
visual magnitudes. Since the early B supergiants are hotter than the rest of the sample, their bolometric corrections and hence their luminosities and, consequently, their masses are larger (see discussion in K08).

Complementary information about the evolutionary status of the targets can be derived from their location on the H-R diagram. With a distance modulus of $\mu=24.93 \pm 0.11 \mathrm{mag}$ as determined in \$5, we have calculated stellar radii and luminosities as described in the previous section. This allows us to position all targets on the H-R diagram in another comparison with the same evolutionary tracks (see Fig. 10, bottom panel). The advantage of using this diagram is that the theoretical dependence of stellar luminosity on mass is very strong and, given the size of the error bars, allows for a more accurate assessment of the original stellar masses. Most obviously, the same objects that appear to be more massive in the previous plot are also the more luminous ones associated with higher-mass evolutionary tracks. This nicely confirms the purely spectroscopic analysis, at least qualitatively.

However, we encounter a problem at lower luminosities. Now there are three objects (instead of just one) located on the $15 M_{\odot}$ track at lower effective temperature. They are No. 2, 4, and 5 in order from lower to higher luminosity. Target No. 2 is particularly suspicious: it has the lowest luminosity of all targets indicating an original mass of less than $15 M_{\odot}$, but on the $\left(\log g, \log T_{\text {eff }}\right)$-plane it has the lowest gravity and effective temperature, resembling an object of significantly higher mass. This indicates a discrepancy between the spectroscopic result and stellar evolutionary path for this specific object.

The discrepancy becomes more apparent when we plot spectroscopic stellar masses against stellar luminosities and compare the results with the corresponding relationship derived from currently available stellar evolutionary tracks at the effective temperatures of early B supergiants and A supergiants (see K08 for a simple fit formula). This is done in Fig. 11, which reveals that the spectroscopic mass of target No. 2 (the object with the lowest mass and luminosity) does not fit on the stellar evolutionary mass-luminosity relationship. Its mass is clearly too low for its present luminosity. We note that K08 in their study of blue supergiants in NGC 300 have found a very similar outlier object (their target No. 17). They speculated that these objects could be stars of lower initial mass now evolving back from the red giant branch to become blue supergiants again at significantly higher luminosity. However, an inspection of the tracks by Meynet \& Maeder (2003) does not support this speculation. None of the tracks with 12,15 , and $20 M_{\odot}$ loops back from the red giant branch, whereas the tracks with significantly lower masses do not reach the luminosity of object No. 2 within their paths. A more attractive explanation appears to be close binary evolution during which No. 2 (and No. 17 of K08) might have lost a significant fraction of their original mass while roughly keeping their original luminosity. Expectedly, such a 
scenario will need to be confirmed by detailed evolutionary calculations. Regardless of the details, it seems that this object has not followed the same evolutionary path as the rest of our sample. It is obvious that the star will not fit into the FGLR defined by regular supergiant stars. Therefore, for distance determination using the FGLR-method we will exclude object No. 2, in the same way K08 excluded their object No. 17.

Fig. 11 seems to indicate that the observed relationship between spectroscopic masses and luminosities for the remaining objects is in slight disagreement with stellar evolution models since most of the objects are somewhat overluminous for their mass. In order to discuss this more quantitatively we use the stellar evolutionary mass-luminosity relationships for A and B supergiants in Fig. 11](see also the fit formulae in K08) to convert observed stellar luminosities into evolutionary masses (as described in \$3). In Fig. 12 we plot the logarithmic ratio of spectroscopic to evolutionary masses as a function of luminosity. As we can see from these plots and Tbl. 4, spectroscopic masses are on average 0.06 dex lower than their evolutionary counterparts (not including No. 2 in calculating the average), indicating indeed a small systematic discrepancy between spectroscopic results and stellar evolution theory. We note that while evolutionary masses have much smaller error bars than spectroscopic masses do due to the steep dependence of luminosity on mass, they might be subjected to systematic uncertainties such as the influence of mass loss over the stellar lifetime or rotationally-induced mixing effects on theoretical luminosities. Of course, spectroscopic masses might be subjected to systematic uncertainties as well, in particular through the way of pressure broadening of the Balmer lines is being accounted for in the process of spectroscopically determining stellar gravities as demonstrated in the previous sections.

In order to assess whether or not the discrepancy encountered is specific to our M33 sample, we repeat the logarithmic ratio of the spectroscopic to evolutionary masses analysis for stars in other galaxies in the bottom panel of Fig. 12. This time we examine targets from the eight galaxies used for the FGLR calibration by K08 and from the most recent study of WLM by Urbaneja et al. (2008). We do not see any indication of a special systematic effect that is only related to M33. The average logarithmic ratio for the 66 targets overplotted is -0.04 dex.

Due to the fact that both evolutionary and spectroscopic masses depend on a presumed luminosity, it is worthwhile to discuss whether or not an inappropriate choice of the distance modulus would cause a systematic effect. Since $M_{\text {spec }} \propto g R^{2} \propto d^{2}, L \propto d^{2}$, and a massluminosity relationship of the form $L \propto M_{\mathrm{evol}}^{\alpha}$, we derive a logarithmic ratio of spectroscopic to evolutionary masses with dependence on $\alpha$ and $d$ as the following:

$$
\log M_{\text {spec }} / M_{\text {evol }}=(2-2 / \alpha) \log d
$$


With $\alpha \sim 3.5$ (see Fig. 11 or K08), we obtain $\log M_{\text {spec }} / M_{\text {evol }}=1.4 \log d$, which means that the distance modulus for M33 would have to be $0.2 \mathrm{mag}$ larger than what we have adopted. As the discussion of the distance to M33 in $\$ 5$ will show, this is unlikely.

\subsection{Metallicity and Metallicity Gradient}

Findings from our quantitative spectroscopic study can be used to assess the metallicity of the young stellar populations in M33 as well as the results of emission line studies of H II regions. Fig. 13 displays the stellar metallicities obtained in consequence as a function of dimensionless angular galactocentric distance. In this, and the corresponding following plots, we use $R_{25}=35.40^{\prime}$ (de Vaucouleurs et al. 1995). Our basic outcome is that the stellar metallicity is close to the solar value at the center and that it decreases by a factor of three at the galactocentric distance of an isophotal radius. A simple linear regression of the form

$$
[Z]=[Z]_{0}+[Z]_{1}\left(R / R_{25}\right)
$$

yields $[Z]_{0}=0.09 \pm 0.04$ dex for the central metallicity and $[Z]_{1}=-0.73 \pm 0.09$ dex $R_{25}^{-1}$ for the angular gradient (equivalent to $-0.07 \pm 0.01 \mathrm{dex} \mathrm{kpc}^{-1}$ for the distance used in this paper). We note, however, that the scatter is significant around $0.5 R_{25}$; it is very likely a real phenomenon rather than an artifact of the uncertainties of our analysis.

Fig. 14 is similar to Fig. 13, but it also includes the H II region Oxygen abundances compiled by Magrini et al. (2007a), H II Neon abundances as determined most recently by Rubin et al. (2008), and Cepheid metallicities as obtained by Beaulieu et al. (2006). (We found that the de-projected galactocentric distances given by Beaulieu et al. (2006) contained errors that we have corrected for Fig. (14). The value for the solar Oxygen abundance adopted to normalize the H II data by Magrini et al. (2007a) is $\log [N(O) / N(H)]+12=8.69$ dex (Allende Prieto et al. 2001). From this sample, we considered only those H II regions for which a determination of the electron temperature was possible. Since the value of the solar Neon abundance seems to be rather uncertain (see discussion in Rubin et al. 2008), we normalize Neon to the average value for B stars in the solar neighborhood and adopt $\log [N(N e) / N(H)]+12=8.08$ dex (Przybilla et al. 2008).

The different data sets seem to converge: they all indicate a significant abundance gradient. Combining both sets of H II region abundances, we derive a slope of $-0.55 \pm 0.09$ $\operatorname{dex} R_{25}^{-1}\left(-0.06 \pm 0.01 \mathrm{dex} \mathrm{kpc}^{-1}\right)$, statistically consistent with the stellar results. With respect to the intercept, a comparison is less robust since several issues could be affecting the zero point of the different abundance scales. The nebular data provide $-0.11 \pm 0.04$ dex, slightly below the stellar result, but nevertheless consistent and not unexpected given the systematics 
that could be affecting both samples (stars and ionized gas). We regard these as consistent results. A least-square fit to all objects in this diagram yields $[Z]_{0}=-0.01 \pm 0.03$ dex for the central value and $[Z]_{1}=-0.71 \pm 0.07 \mathrm{dex} R_{25}^{-1}$ for the gradient. Within the uncertainties, this agrees with the results obtained from the supergiant stars alone. We note again that the results for the H II regions displayed in this figure also indicate a large scatter around the regression curve.

This significant metallicity gradient is in obvious disagreement with the result found by Rosolowsky \& Simon (2008), who carried out a comprehensive study of H II regions in their M33 metallicity project. Fig. 15 compares the Oxygen abundances of our supergiants with that of their H II regions. While our data points largely coincide with theirs at large galactocentric distances, Rosolowsky \& Simon (2008) find many H II regions with very low oxygen abundance in the central region of M33 for which we have no stellar counterparts in our sample. At this point, we have no explanation for this discrepancy.

\section{Distance}

In this section we employ two different methods to determine the distance to M33. First, we use the stellar parameters from this spectroscopic study and apply the FGLR method; second, we analyse Hubble Space Telescope (HST) Advanced Camera for Surveys (ACS) photometry of three outer fields of M33 and obtain a distance from the $I$-band magnitude of the tip of the red giant branch (TRGB method). The results from each technique will be discussed accordingly.

\subsection{FGLR and Distance to M33}

The FGLR is a tight correlation between the flux-weighted gravity $\left(g_{F} \equiv g / T^{4}{ }_{\text {eff }}\right)$ and the absolute magnitude of BA supergiants. The physical background, detection and the calibration in nearby galaxies has been described in Kudritzki et al. (2003) and K08. Urbaneja et al. (2008) were the first to use the FGLR for distance determination and found a distance modulus of 24.99 mag for the metal-poor dwarf galaxy WLM, in good agreement with most recent TRGB distance determinations. Here, we follow the same procedure as was detailed in Urbaneja et al. (2008).

The FGLR has the form

$$
M_{\mathrm{bol}}=a\left(\log g_{F}-1.5\right)+b
$$


with the most recent calibration provided by K08, $a=3.41$ and $b=-8.02$. Our spectroscopic analysis provides de-reddened apparent bolometric magnitude $m_{\text {bol }}$ (see calculation details in $\S 3.4$ ) and flux-weighted gravity for each of our targets (except for object No. 2, as explained in $§ 4.2)$ that we can fit with a regression of the form

$$
m_{\mathrm{bol}}=a_{\mathrm{M} 33}\left(\log g_{F}-1.5\right)+b_{\mathrm{M} 33} .
$$

The fit result is shown in Fig. 16 (top). Since our M33 targets span only a limited range in $g_{F}$ compared to the K08 sample, we adopted the slope value provided by K08 (letting $\left.a_{\mathrm{M} 33}=a\right)$ and fit only $b_{\mathrm{M} 33}$. The difference between $b$ and $b_{\mathrm{M} 33}$ yields the distance modulus, which we determine to be $\mu=24.93 \pm 0.11 \mathrm{mag}$ (the error is calculated similarly as in Urbaneja et al. 2008). Fig. 16 (bottom) then compiles the FGLR results for our M33 targets using this distance modulus as well as that for 9 other galaxies from K08 and Urbaneja et al. (2008). The plot does not indicate any systematic differences between the M33 sample and the remaining sample.

We note that the present calibration of the FGLR by K08 is based on a sample of supergiants selected from NGC 300 and seven Local Group galaxies for which distances were adopted. A cleaner, more accurate and systematic method to calibrate the relationship would be to use a large sample of blue supergiants in the Large Magellanic Clouds (LMC). This work is presently under way.

\subsection{TRGB and Distance to M33}

We use observations of M 33 available through the HST archive to measure a distance based on photometry of stars at the TRGB. The footprints of the observations taken with ACS are shown in Fig. 17 (top) as yellow outlines. The two fields towards the east (left) side of the image are from HST Program 9479's study of M33's halo; these fields are combined in the ensuing analysis. The remaining field to the south of the image comes from HST Program 10190's observations of the outer disk. We choose these sets of images from among the many available through the HST archive in order to balance the need of minimizing issues of stellar crowding and reddening within M33 with the need for a well-populated RGB.

Both programs use the $F 814 W$ and $F 606 W$ filters, which can be described as "wide $I$ ' and 'wide $V$ ' filters, respectively. We perform photometry and conduct artificial star tests on the images using the DOLPHOT software package, which is a modified version of HSTphot (Dolphin 2000). The resulting color-magnitude diagrams are presented in Fig. 17 (bottom). Using the real and artificial photometry we measure the $F 814 W$ magnitude and

color of the TRGB using a maximum-likelihood method described by Makarov et al. (2006). 
The $F 814 W$ magnitude of the TRGB measured in the halo fields from Program 9479 is $20.92_{-0.11}^{+0.09}$ with $F 606 W-F 814 W=1.35 \pm 0.05$, while the observations of the disk from Program 10190 yields $20.89_{-0.05}^{+0.09}$ with $F 606 W-F 814 W=1.47_{-0.08}^{+0.02}$; the TRGB magnitudes are marked with broken horizontal lines in Fig. 17. The flux from stars at the TRGB is least sensitive to age and metallicity in the $I$-band, and has an absolute magnitude of $M_{I} \approx-4.05$ (Rizzi et al. 2007). Rizzi et al. (2007) also describe a method for calculating a distance modulus that includes a zero-point calibration and metallicity correction; the formula for which we reproduce below:

$$
D M=m_{\mathrm{TRGB}}-A_{F 814 W}+4.06-0.20\left[(F 606 W-F 814 W)-\left(A_{F 606 W}-A_{F 814 W}\right)-1.23\right],
$$

where $m_{\mathrm{TRGB}}$ is the apparent magnitude in $F 814 W$, and $A_{F 814 W}$ and $A_{F 606 W}$ are foreground extinction for the two filters, respectively. We account for foreground reddening using the dust maps of Schlegel et al. (1998) with a value of $E(B-V)=0.04$ mag and their prescription for converting to HST flight magnitude. Whilst we have encountered much higher reddening values in our study of the blue supergiants, the use of only foreground reddening is very likely justified in these outer fields. Combining these corrections with the measured magnitudes of the tip produces distance moduli of $\mu=24.86_{-0.13}^{+0.09} \mathrm{mag}$ for the halo observations and $\mu=24.82_{-0.06}^{+0.10}$ for with the outer disk field. As an average TRGB distance modulus we adopt $\mu=24.84 \pm 0.10 \mathrm{mag}$, which is in basic agreement with that found by Rizzi et al. (2007) using HST WFPC2 data $(\mu=24.71 \pm 0.04)$.

\subsection{Discussion}

The two independent methods described in this paper, the FGLR and the TRGB, both yield a large distance modulus and agree with each other within statistical uncertainties. The FGLR-method stands unaffected by interstellar extinction uncertainties because reddening has been determined for each target individually through quantitative spectral analysis, which yields accurate stellar parameters and, thus, intrinsic SEDs and colors. In this regard, the FGLR work presented here is very similar to the quantitative spectral analysis of the detached eclipsing O-star binary D33 J1013346.2+304439.9 by Bonanos et al. (2006), who obtained a distance modulus of $\mu=24.92 \pm 0.12$ mag with a reddening of $E(B-V)=0.09$ mag.

$\mathrm{B}$ and A supergiants as well as $\mathrm{O}$ stars belong to the young stellar population in the dust-obscured disk of M33. An independent and accurate determination of extinction hence falls inevitably from their use as distance indicators. The same is also true for other types of objects belonging to a population of similar age, such as Long Period Variables and 
Cepheids. For the former, Pierce et al. (2000) have carried out a careful photometric study of the period-absolute magnitude relationship of LPVs in Per OB1, the LMC, and M33 using $R$ and $I$ photometry and a narrow band filter at $8250 \AA$ (particularly to correct for the varying strength of $\mathrm{TiO}$ absorption). Assuming a reddening value of $E(B-V)=0.1$ mag for M33, they obtained a distance modulus of $\mu=24.85 \pm 0.13$ mag to the galaxy. While reddening was not determined for individual targets, the value assumed is close to the average value of $0.083 \mathrm{mag}$ found in our study. Thus, it seems consistent that their distance modulus is also in agreement with ours.

The situation with Cepheid distance determinations turns out to be more complex. Based on HST WFPC2 photometry from Freedman et al. (1991) and Lee et al. (2002), the distance modulus was found to be $\mu=24.62 \pm 0.15$ mag and $\mu=24.52 \pm 0.14$ (random) \pm 0.13 (systematic) mag, respectively. Whilst the former study applied a correction for metallicity, the latter did not. Both works considered a very high value of reddening, $E(B-$ $V)=0.20 \mathrm{mag}$, which was derived from the difference in the apparent distance moduli between $V$ - and $I$-bands. This reddening value differs substantially from the average value of 0.08 mag found for the B and A supergiants in our study.

Since Cepheids are also young massive stars (though less massive than blue supergiants), they are found at similar sites as are blue supergiants, and thus, the reason why their reddening is systematically higher is unclear. The direct determination of reddening from spectroscopy should be superior to the indirect way of using the difference of apparent distance moduli in $V$ - and $I$-bands. We note that the latter must also depend on metallicity (an effect that had not been considered in the pertinent studies) as well as an assumed value of reddening for the calibration sample of Cepheids in the LMC. If one assumes that the value of $E(B-V)=0.2 \mathrm{mag}$ as adopted for the Cepheids study is too high and uses the average value of $0.083 \mathrm{mag}$ from the blue supergiants study instead, then the Cepheid distance modulus would increase by $0.37 \mathrm{mag}$, yielding a distance in excellent agreement with our FGLR and TRGB values. This drastic improvement illustrates two things: first, the HST observations of Cepheids seem to be compatible with a large distance modulus; second and most importantly, the accurate elimination of the reddening uncertainty is absolutely crucial for the determination of distances using Cepheids.

Very recently, Scowcroft et al. (2009) presented ground-based $B-, V$-, $I$-band photometry of Cepheids in the center of M 33 and in the southern spiral arm, some 4 to 5 kpc away from the center, with 91 and 28 objects in the two fields, respectively. The use of the Wesenheit $W_{V I}$ magnitude, which was assumed to be reddening-free, yielded largely discrepant distance moduli in the center and in the spiral arm (24.37 and 24.54 mag, respectively) that the authors attributed to a metallicity difference between the regions. They derived a true 
distance modulus of $\mu=24.53 \pm 0.11$ mag accordingly, very much in agreement with the HST photometry Cepheid papers discussed in the previous paragraph. The paper does not provide an estimate of $E(B-V)$, but given their distance agreement with the published HST Cepheid work, we assume that their reddening value would be close to $0.2 \mathrm{mag}$. We also note that, at least from the basic principle, the use of the Wesenheit magnitude is very similar to the use of difference between apparent distance moduli obtained in $V$ - and $I$-bands. Therefore, we feel that the same discussion on reddening and distance determination in the previous paragraphs applies here as well. (This distance modulus is based on a distance modulus of 18.40 mag for the LMC, while our FGLR calibration adopted 18.50 mag. Thus, it would increase by $0.1 \mathrm{mag}$ if our distance were assumed.)

It is also important to take into account that the metallicity difference between the two fields studied by Scowcroft et al. (2009) seems to be significantly smaller than the value of 0.566 dex they adopted. This value was obtained based on the assumption of a twocomponent slope as originally proposed by Vilchez et al. (1988) and then also adopted as one possibility by Magrini et al. (2007b). However, as discussed in $\$ 4.3$ and shown in our Fig. 14, both the metallicities from the stars and from H II regions do not support a very steep gradient towards the center of M 33. Using our result from $\$ 4.3$ for the abundance gradient, the metallicity difference between the two Scowcroft et al. fields is only 0.31 dex. With this number, the dependence of the Cepheid distance moduli on logarithmic metallicity changes would become $\gamma=0.55 \mathrm{mag} \mathrm{dex}^{-1}$, almost twice as high as the value of 0.29 found by Macri et al. (2006) and that derived by Scowcroft et al. (2009).

There were also a number of other distance determinations methods in the literature that involved stars of significantly older populations. The TRGB method, as applied in this work, is a typical example. Our distance modulus agrees very well with that of Kim et al. (2002), who used HST WFPC photometry in 10 fields and obtained $\mu=24.81 \pm 0.13$ mag. Note also that Kim et al. (2002) were the first to draw attention to the fact that the shorter distance modulus associated with Cepheid distances might be a result of overestimating of interstellar reddening.

In addition, three studies involving ground-based photometry were published later yielding quite a range in distance: McConnachie et al. (2004), $\mu=24.50 \pm 0.06$ mag; Galleti et al. (2004), $\mu=24.64 \pm 0.15 \mathrm{mag}$; and Tiede et al. (2004), $\mu=24.69 \pm 0.07$ mag. It seems that systematic effects such as $I$-band calibration and the algorithm used for TRGB edge detection are of importance when comparing these results. We note, however, that two of these studies agree with the results acquired with HST photometry within the corresponding error margins.

Other independent investigations using older stellar populations were carried out by Sarajedini et al. 
(2000) and Ciardullo et al. (2004) and confirmed a large distance modulus. The former studied Horizontal Branch stars and found $\mu=24.84 \pm 0.16 \mathrm{mag}$, whereas the latter used the Planetary Nebulae Luminosty Function to obtain $\mu=24.86_{-0.11}^{+0.07}$ mag.

Sarajedini et al. (2006) used HST ACS photometry of RR Lyrae field stars because at minimum light, the intrinsic $V-I$ color of RR Lyrae stars is well defined and independent of metallicity and period. This allows for an estimate of reddening from the construction of $V$ and $I$ light curves. Unfortunately, the reddening determination was somewhat uncertain and 18 stars in their sample turned out to have negative reddening. The authors found $\mu=24.67$ \pm 0.07 mag while excluding those objects, and $\mu=24.76 \pm 0.08$ mag with them included. We believe that these 18 stars are simply cases with very small reddening and represent the uncertainty of the procedure; thus, their inclusion is justified. This, then, exhibits agreement with the HST TRGB results as well as with that derived from the HB and PN stars.

In summary, we conclude that a large distance modulus as obtained with the FGLR and TRGB work presented here is well supported by the value of reddening we found and agrees well with other independent work based on low-mass stars of older populations.

\section{Conclusions}

Motivated by the need to achieve precision in extragalactic distance determination and to resolve the large discrepancy in the distance modulus to the nearby Triangulum Galaxy M33, we undertake an independent investigation applying the flux-weighted gravityluminosity relationship to a sample of 22 blue supergiants at various galactocentric distances in M33. With medium resolution spectra from Keck, we carry out a quantitative spectral analysis and measured stellar parameters such as rotational velocities, stellar gravities, effective temperatures, and metallicities. Together with state-of-the-art non-LTE model atmospheres spanning an expansive grid in $\left(T_{\text {eff }}, \log g\right)$ parameter space and photometry available in literature, we further derive interstellar reddening, stellar luminosities, radii, and masses. The combinations of these parameters allow us to determine a distance modulus of $24.93 \pm 0.11 \mathrm{mag}$ to M33.

We discuss in detail the stellar properties of our supergiants in relation to their predicted evolutionary paths. On the high-luminosity end, our spectroscopic results are nicely confirmed by the evolutionary tracks on the H-R diagram. On the low-luminosity end, however, we observe one object (similar to the one found by K08) that appears to follow a different path and offer close binary evolution as an explanation, which will require further confirmation from detailed evolutionary calculations in the future. Furthermore, we note a 
small systematic discrepancy between spectroscopic and evolutionary masses, however it is unlikely to be caused by uncertainties in the distance modulus. Our discussion on metallicities specifies the significant metallicity gradient as a function of galactocentric distance that we find in M33, with large scatter about the regression curve. This is similar to the results of several other metal abundance studies of H II regions in literature.

Our FGLR for M33 follows that for other galaxies very nicely, giving confidence to both the reliability of its calibration and the distance modulus thus determined. Using HST photometry available in the archive, we also present a TRGB distance modulus that nicely converges with our FGLR result within the uncertainties. These distances agree well with other published large distance moduli and low reddening values found in literature, based on work on detached eclipsing binary, long period variables, horizontal branch stars, and planetary nebulae luminosity function. The discrepancy between our results and that from Cepheid studies can be explained by differences in interstellar reddening assumed in the different work, and we point out that the Cepheid distance moduli will increase if the reddening value and metallicity were properly determined and applied. However, we note that a final conclusion will only be possible after a more rigorous calibration of the FGLR using a large sample of LMC blue supergiants.

The data presented herein were obtained at the W.M. Keck Observatory, which is operated as a scientific partnership among the California Institute of Technology, the University of California and the National Aeronautics and Space Administration. The Observatory was made possible by the generous financial support of the W.M. Keck Foundation. The authors wish to recognize and acknowledge the very significant cultural role and reverence that the summit of Mauna Kea has always had within the indigenous Hawaiian community. We are most fortunate to have the opportunity to conduct observations from this mountain.

\section{REFERENCES}

Allende Prieto, C., Lambert, D. L., \& Asplund, M. 2001, ApJ, 556, L63

Beaulieu, J.-P., Buchler, J. R., Marquette, J.-B., Hartman, J. D., \& Schwarzenberg-Czerny, A. 2006, ApJ, 653, L101

Bonanos, A. Z., et al. 2006, ApJ, 652, 313

Bresolin, F., Kudritzki, R.-P., Mendez, R. H., \& Przybilla, N. 2001, ApJ, 548, L159 
Brunthaler, A., Reid, M. J., Falcke, H., Greenhill, L. J., \& Henkel, C. 2005, Science, 307, 1440

Cardelli, J. A., Clayton, G. C., \& Mathis, J. S. 1989, ApJ, 345, 245

Ciardullo, R., Durrell, P. R., Laychak, M. B., Herrmann, K. A., Moody, K., Jacoby, G. H., \& Feldmeier, J. J. 2004, ApJ, 614, 167

de Vaucouleurs, G., de Vaucouleurs, A., Corwin, H. G., Buta, R. J., Paturel, G., \& Fouque, P. 1995, VizieR Online Data Catalog, 7155, 0

Dolphin, A. E. 2000, PASP, 112, 1383

Freedman, W. L., Wilson, C. D., \& Madore, B. F. 1991, ApJ, 372, 455

Frieman, J. A., Turner, M. S., \& Huterer, D. 2008, ARA\&A, 46, 385

Galleti, S., Bellazzini, M., \& Ferraro, F. R. 2004, A\&A, 423, 925

Gieren, W., Pietrzyński, G., Soszyński, I., Bresolin, F., Kudritzki, R.-P., Minniti, D., \& Storm, J. 2005, ApJ, 628, 695

Gray, D. F. 1992, The Observation and Analysis of Stellar Photospheres (The Observation and Analysis of Stellar Photospheres, by David F. Gray, pp. 470. ISBN 0521408687. Cambridge, UK: Cambridge University Press, June 1992.)

Howarth, I. D. 2004, in IAU Symposium, Vol. 215, Stellar Rotation, ed. A. Maeder \& P. Eenens, 33-+

Humphreys, R. M., \& Sandage, A. 1980, ApJS, 44, 319

Ivanov, G. R., Freedman, W. L., \& Madore, B. F. 1993, ApJS, 89, 85

Kim, M., Kim, E., Lee, M. G., Sarajedini, A., \& Geisler, D. 2002, AJ, 123, 244

Kudritzki, R. P., Bresolin, F., \& Przybilla, N. 2003, ApJ, 582, L83

Kudritzki, R.-P., Urbaneja, M. A., Bresolin, F., Przybilla, N., Gieren, W., \& Pietrzynski, G. 2008, Astrophysical Journal, 681, 269

Lee, M. G., Kim, M., Sarajedini, A., Geisler, D., \& Gieren, W. 2002, ApJ, 565, 959

Lennon, D. J. 1997, A\&A, 317, 871

Macri, L. M., Stanek, K. Z., Bersier, D., Greenhill, L. J., \& Reid, M. J. 2006, ApJ, 652, 1133 
Maeder, A., \& Meynet, G. 2005, A\&A, 440, 1041

Magrini, L., Corbelli, E., \& Galli, D. 2007a, A\&A, 470, 843

Magrini, L., Vílchez, J. M., Mampaso, A., Corradi, R. L. M., \& Leisy, P. 2007b, A\&A, 470, 865

Makarov, D., Makarova, L., Rizzi, L., Tully, R. B., Dolphin, A. E., Sakai, S., \& Shaya, E. J. 2006, AJ, 132, 2729

Marinoni, C., Davis, M., Coil, A. L., \& Finkbeiner, D. 2001, ArXiv Astrophysics e-prints

Massey, P., Armandroff, T. E., Pyke, R., Patel, K., \& Wilson, C. D. 1995, AJ, 110, 2715

Massey, P., Bianchi, L., Hutchings, J. B., \& Stecher, T. P. 1996, ApJ, 469, 629

Massey, P., Olsen, K. A. G., Hodge, P. W., Strong, S. B., Jacoby, G. H., Schlingman, W., \& Smith, R. C. 2006, AJ, 131, 2478

McConnachie, A. W., Irwin, M. J., Ferguson, A. M. N., Ibata, R. A., Lewis, G. F., \& Tanvir, N. 2004, MNRAS, 350, 243

Meynet, G., \& Maeder, A. 2003, A\&A, 404, 975

Pierce, M. J., Jurcevic, J. S., \& Crabtree, D. 2000, MNRAS, 313, 271

Przybilla, N., Butler, K., Becker, S. R., \& Kudritzki, R. P. 2006, A\&A, 445, 1099

Przybilla, N., Nieva, M.-F., \& Butler, K. 2008, ApJ, 688, L103

Puls, J., Urbaneja, M. A., Venero, R., Repolust, T., Springmann, U., Jokuthy, A., \& Mokiem, M. R. 2005, A\&A, 435, 669

Rizzi, L., Tully, R. B., Makarov, D., Makarova, L., Dolphin, A. E., Sakai, S., \& Shaya, E. J. 2007, ApJ, 661, 815

Rosolowsky, E., \& Simon, J. D. 2008, ApJ, 675, 1213

Rubin, R. H., et al. 2008, MNRAS, 387, 45

Sarajedini, A., Barker, M. K., Geisler, D., Harding, P., \& Schommer, R. 2006, AJ, 132, 1361

Sarajedini, A., Geisler, D., Schommer, R., \& Harding, P. 2000, AJ, 120, 2437

Schiller, F., \& Przybilla, N. 2008, A\&A, 479, 849 
Schlegel, D. J., Finkbeiner, D. P., \& Davis, M. 1998, ApJ, 500, 525

Scowcroft, V., Bersier, D., Mould, J. R., \& Wood, P. R. 2009, MNRAS, 635

Sheinis, A. I., Bolte, M., Epps, H. W., Kibrick, R. I., Miller, J. S., Radovan, M. V., Bigelow, B. C., \& Sutin, B. M. 2002, PASP, 114, 851

Tiede, G. P., Sarajedini, A., \& Barker, M. K. 2004, AJ, 128, 224

Urbaneja, M. A., et al. 2005a, ApJ, 622, 862

Urbaneja, M. A., Herrero, A., Kudritzki, R.-P., Najarro, F., Smartt, S. J., Puls, J., Lennon, D. J., \& Corral, L. J. 2005b, ApJ, 635, 311

Urbaneja, M. A., Kudritzki, R.-P., Bresolin, F., Przybilla, N., Gieren, W., \& Pietrzyński, G. 2008, ApJ, 684, 118

Vilchez, J. M., Pagel, B. E. J., Diaz, A. I., Terlevich, E., \& Edmunds, M. G. 1988, MNRAS, 235,633 

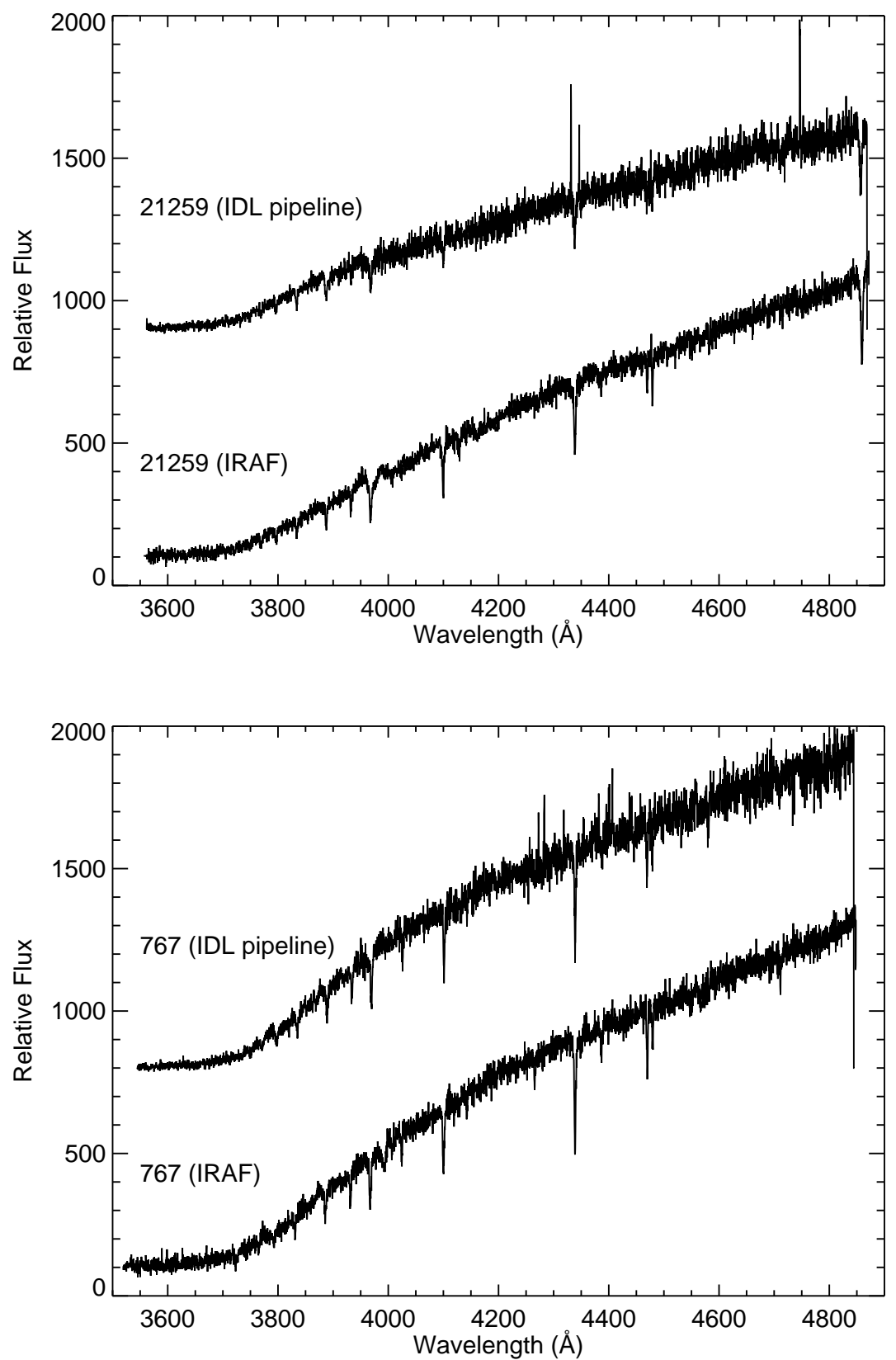

Fig. 1.- Comparison of pre-normalized flux spectra for two selected supergiants of late (top) and early (bottom) B types reduced by DEEP2 IDL pipeline and by IRAF reduction packages, respectively. Notice that the SNR in this blue wavelength range is much better for the IRAF-reduced spectra than for the pipeline results. 


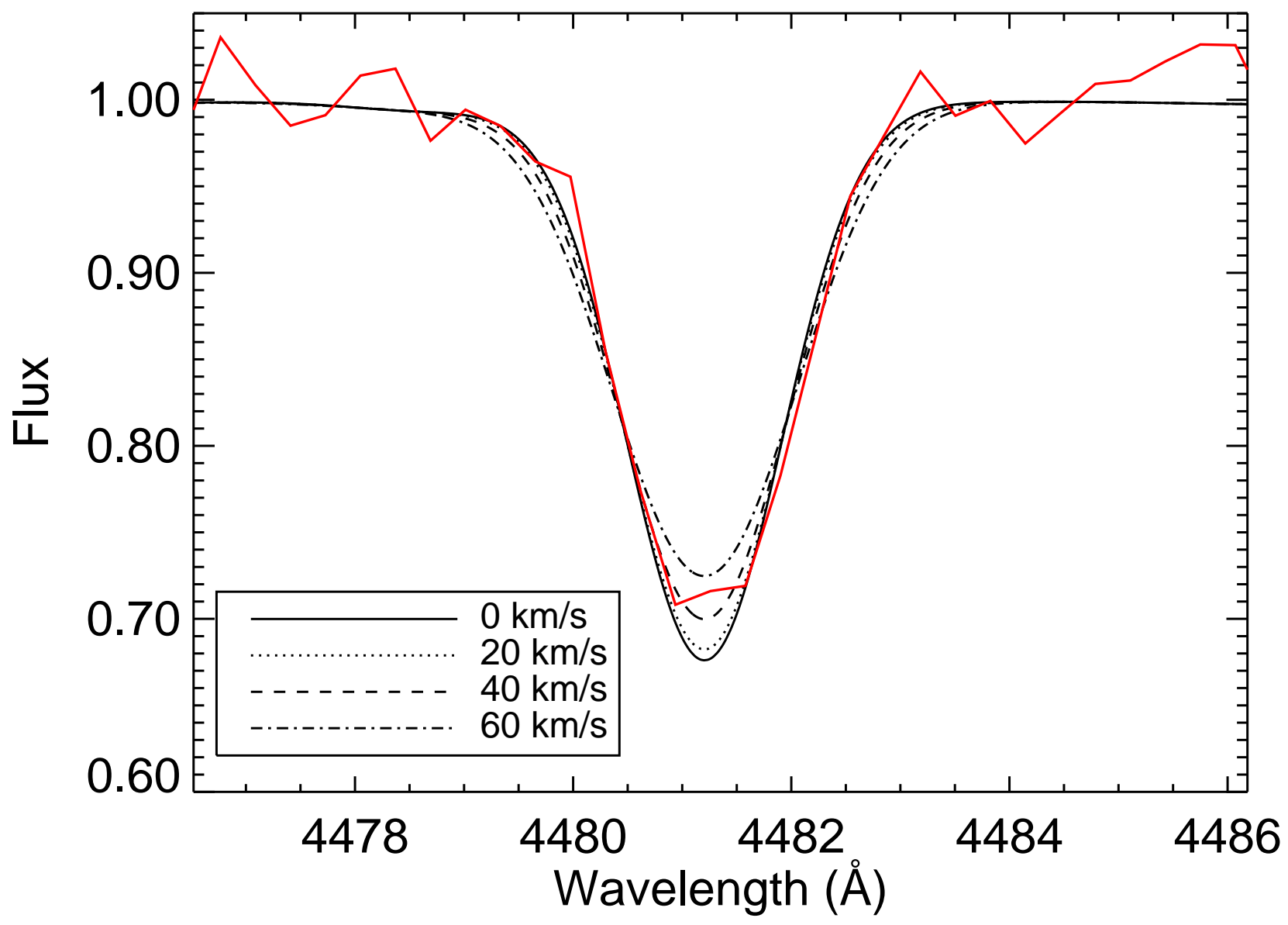

Fig. 2.- The effects of different rotational velocities on the shape of the Mg II (4481) line. As the velocity increases, the line grows flatter and flatter, with more pronounced effects in the core than in the wings. 

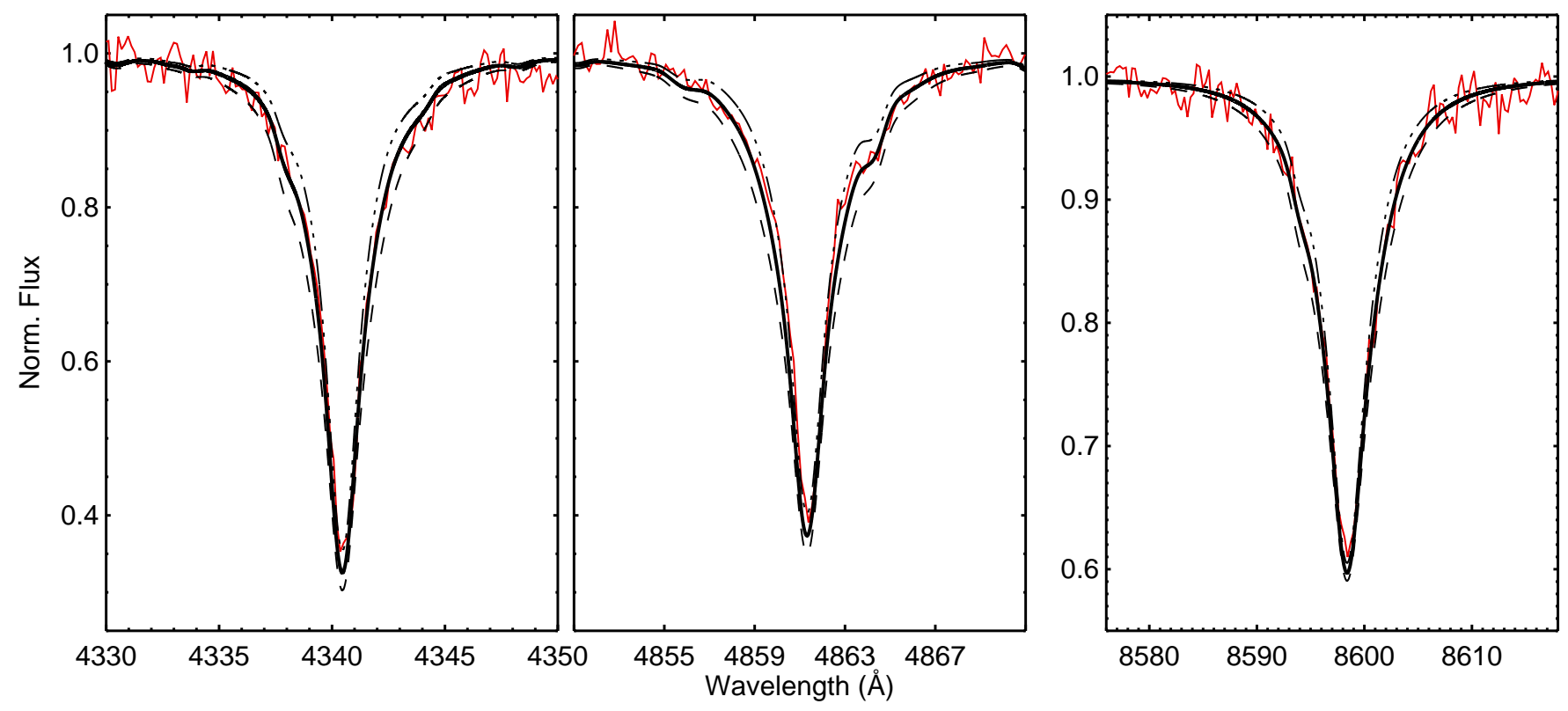

Fig. 3.- These panels show the Balmer lines fit (from left to right: $\mathrm{H}_{\gamma}, \mathrm{H}_{\beta}, \mathrm{H}_{3-14}$ ) for the object No.11. The observed spectrum (thin) is overplotted with the model (thick) with $T_{\text {eff }}$ of $9600 \mathrm{~K}$ and $\log g$ at $1.40_{-0.10}^{+0.10}$ dex where the errors are represented by dashed lines. 

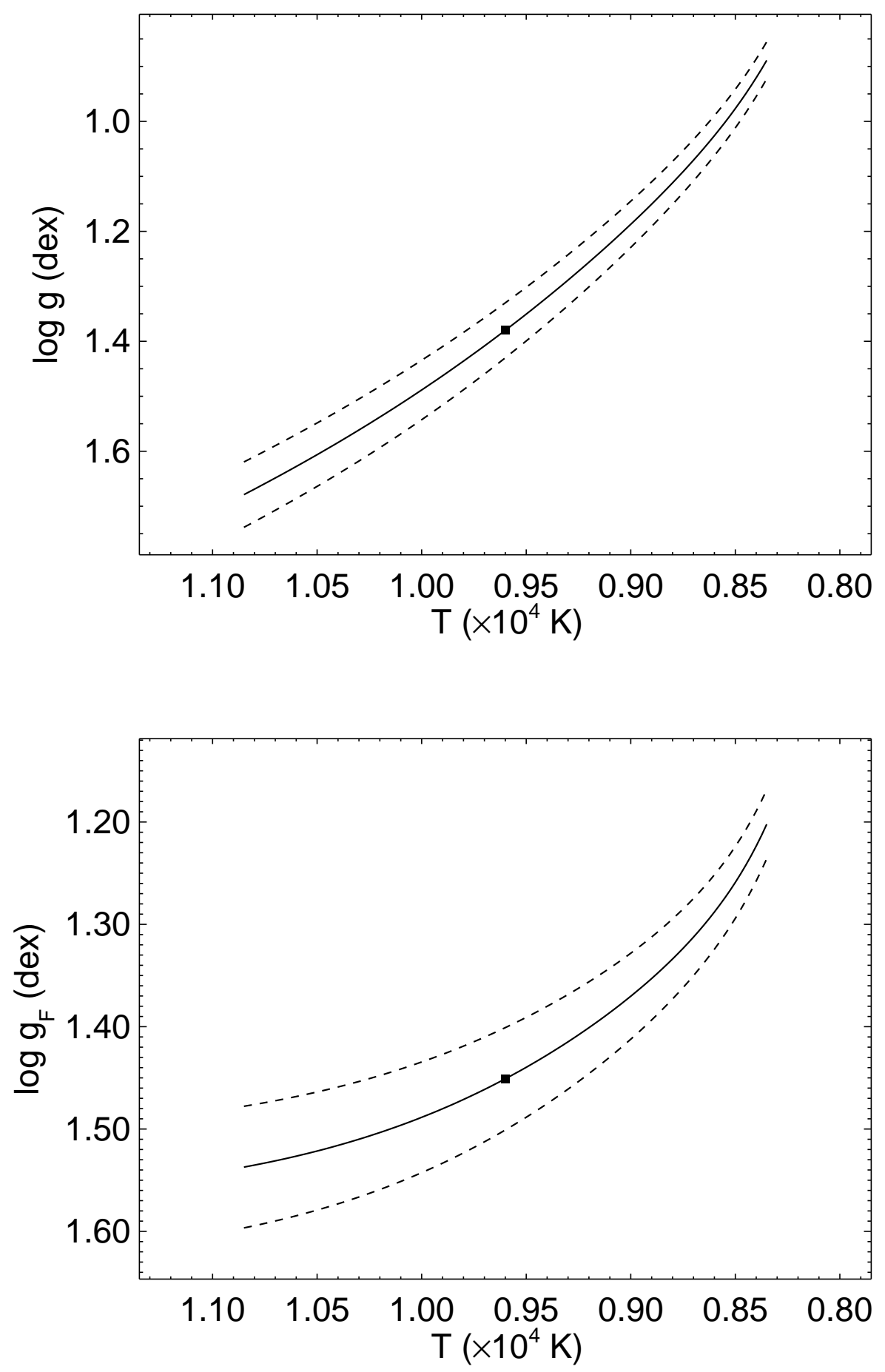

Fig. 4.- Balmer line fit curve for object No. 11 in the log $g-T_{\text {eff }}$ plane (top) and the $\log g_{F^{-}}$ $T_{\text {eff }}$ plane (bottom). The dashed curves correspond to the maximum fitting errors. 


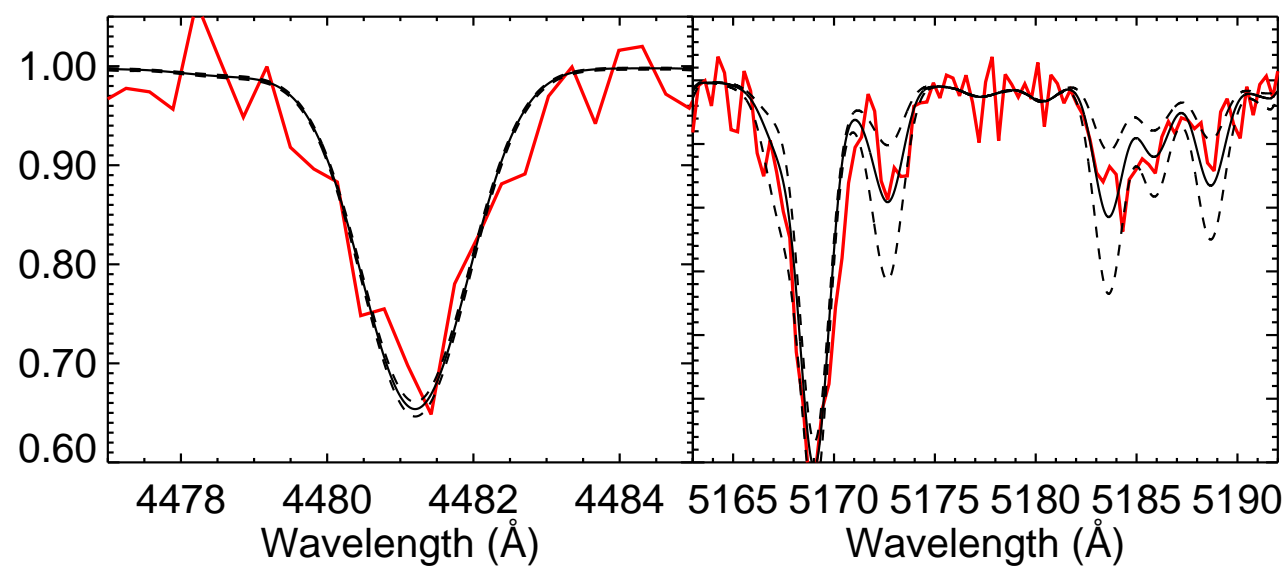

Fig. 5.- Fit of the Mg I/II ionization equilibrium along the Balmer line fit curve of object No. 5. Three models are overplotted. $T_{\text {eff }}=8750 \mathrm{~K}$ and $\log g=1.30$ (solid curve), $T_{\text {eff }}$ $=8300 \mathrm{~K}$ and $\log g=1.05$ and $T_{\text {eff }}=9250 \mathrm{~K}$ and $\log g=1.50$ (both dashed). While the $\mathrm{Mg}$ II line at $4481 \AA$ is insensitive to model parameter changes along the fit curve in this temperature range and depends only on metallicity, the $\mathrm{MgI}$ lines (vertical bars at the right hand figure) show a very strong temperature dependence. A temperature of $T_{\text {eff }}=8750 \pm 250$ is obtained from this plot. 

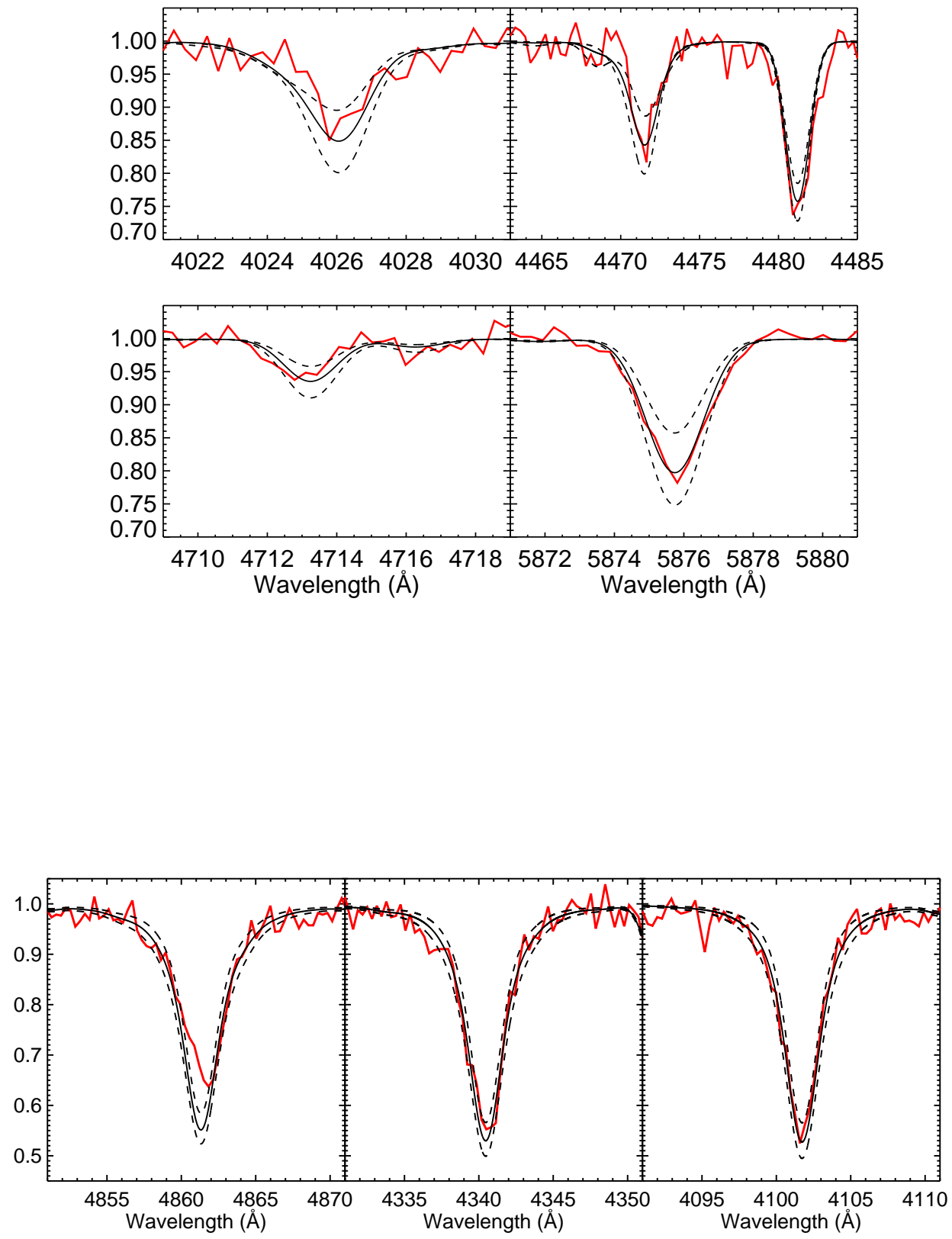

Fig. 6. - Line fit of object No. 1. Top: the HeI lines at $4026 \AA, 4471 \AA, 4713 \AA$, and $5876 \AA$ along the Balmer line fit curve of object No. 1. Three models are overplotted. $T_{\text {eff }}=$ $11000 \mathrm{~K}$ and $\log g=1.55$ (solid curve), $T_{\text {eff }}=10000 \mathrm{~K}$ and $\log g=1.35$ and $T_{\text {eff }}=12000 \mathrm{~K}$ and $\log g=1.70$ (both dashed). Bottom: Balmer lines (from left to right: $\mathrm{H}_{\beta}, \mathrm{H}_{\gamma}, \mathrm{H}_{\delta}$ ) at $T_{\text {eff }}=11000 \mathrm{~K}$. The gravities are $\log g=1.55$ (solid) and 1.45 and 1.65 (both dashed), respectively. Note that $\mathrm{H}_{\beta}$ is affected by stellar wind emission in the line core. 


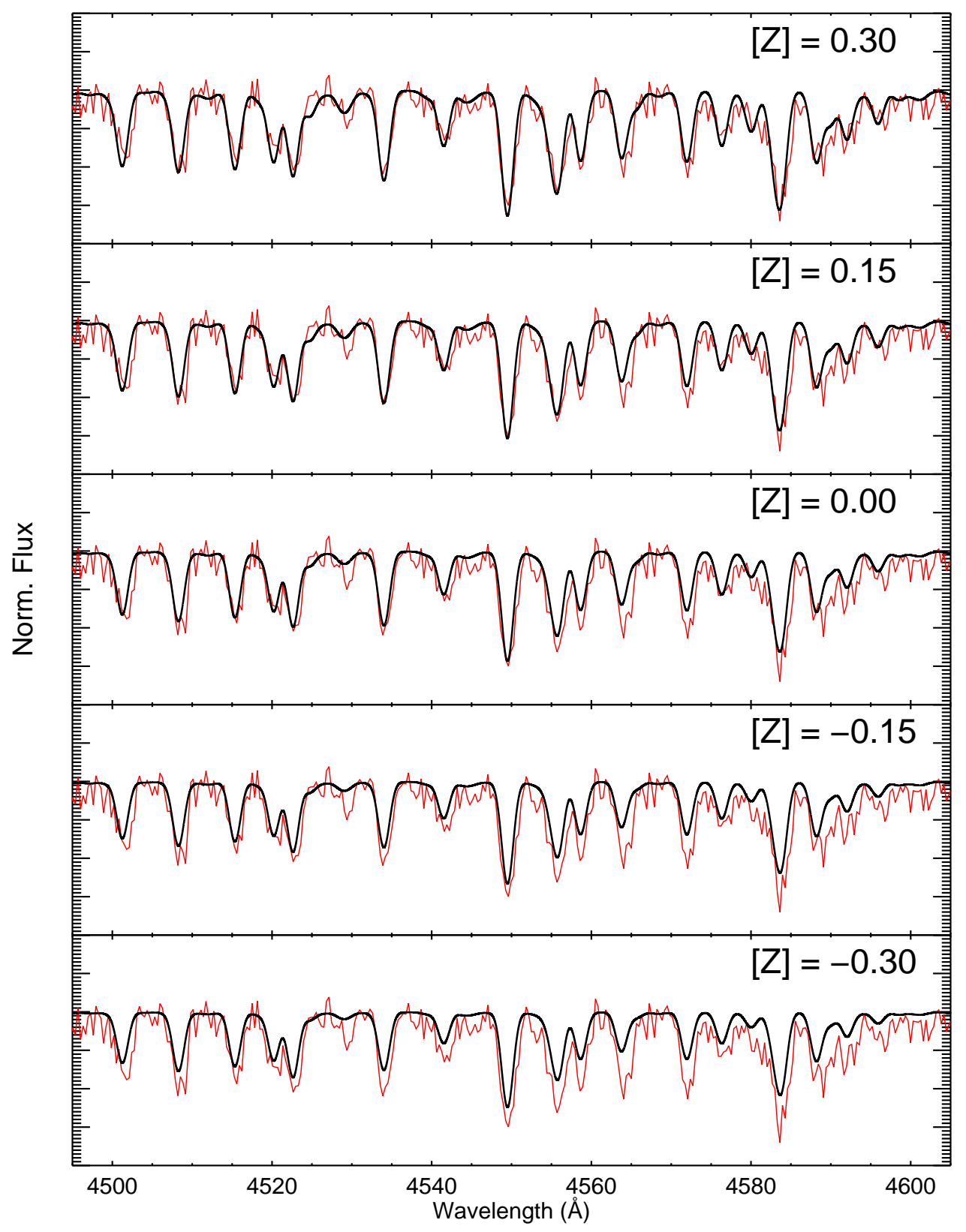

Fig. 7.- These panels show the metal line fit for the object No. 5. The observed spectrum (thin) is overplotted with the model (thick) with $T_{\text {eff }}=8750 \mathrm{~K}$ and $\log g=1.30$ dex at increasing metallicities. The best-fit metallicity is $\log \left(Z / Z_{\odot}\right)=0.15$ (second row). 


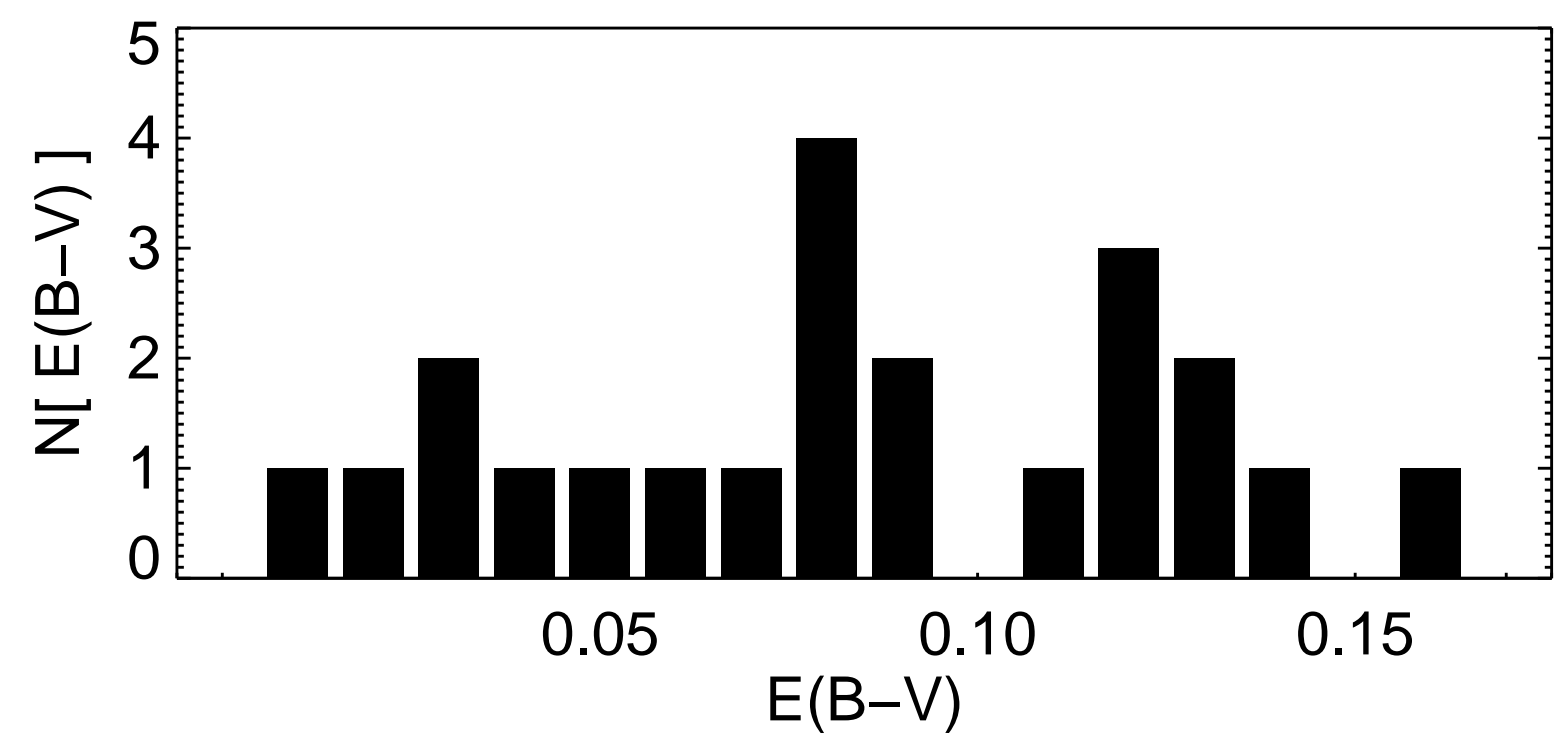

Fig. 8. - The distribution of reddening $E(B-V)$ for our B and A supergiants; the average $<E(B-V)>$ for our sample is $0.083 \mathrm{mag}$. 


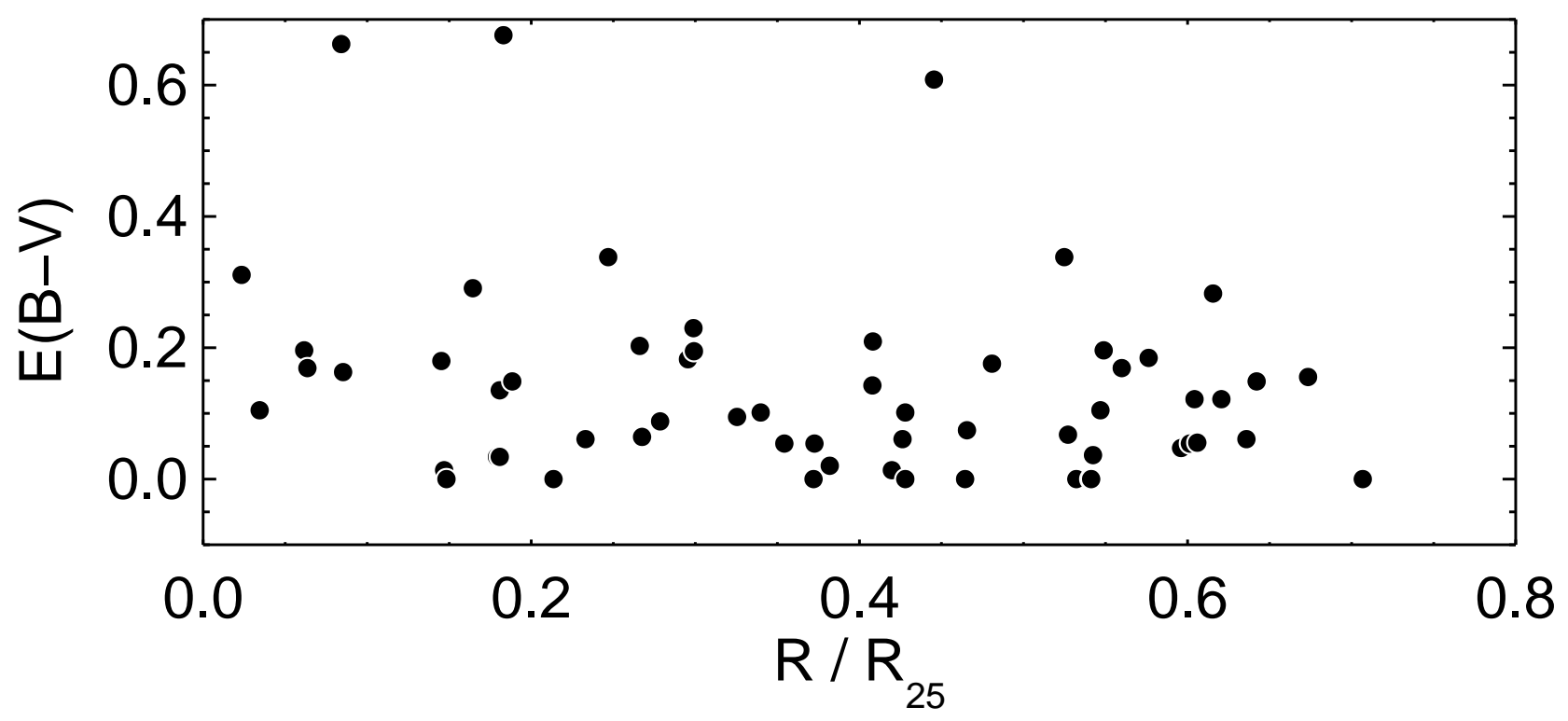

Fig. 9.- Reddening $E(B-V)$ of $\mathrm{M} 33 \mathrm{H}$ II regions as a function of angular galactic distance $\left(R_{25}=35.40^{\prime}\right.$; de Vaucouleurs et al. 1995). The data have been taken from Rosolowsky \& Simon (2008), see text. The average $\langle E(B-V)\rangle$ for this sample is 0.11 mag (not including the three extreme objects with reddening larger than $0.6 \mathrm{mag}$ ). 

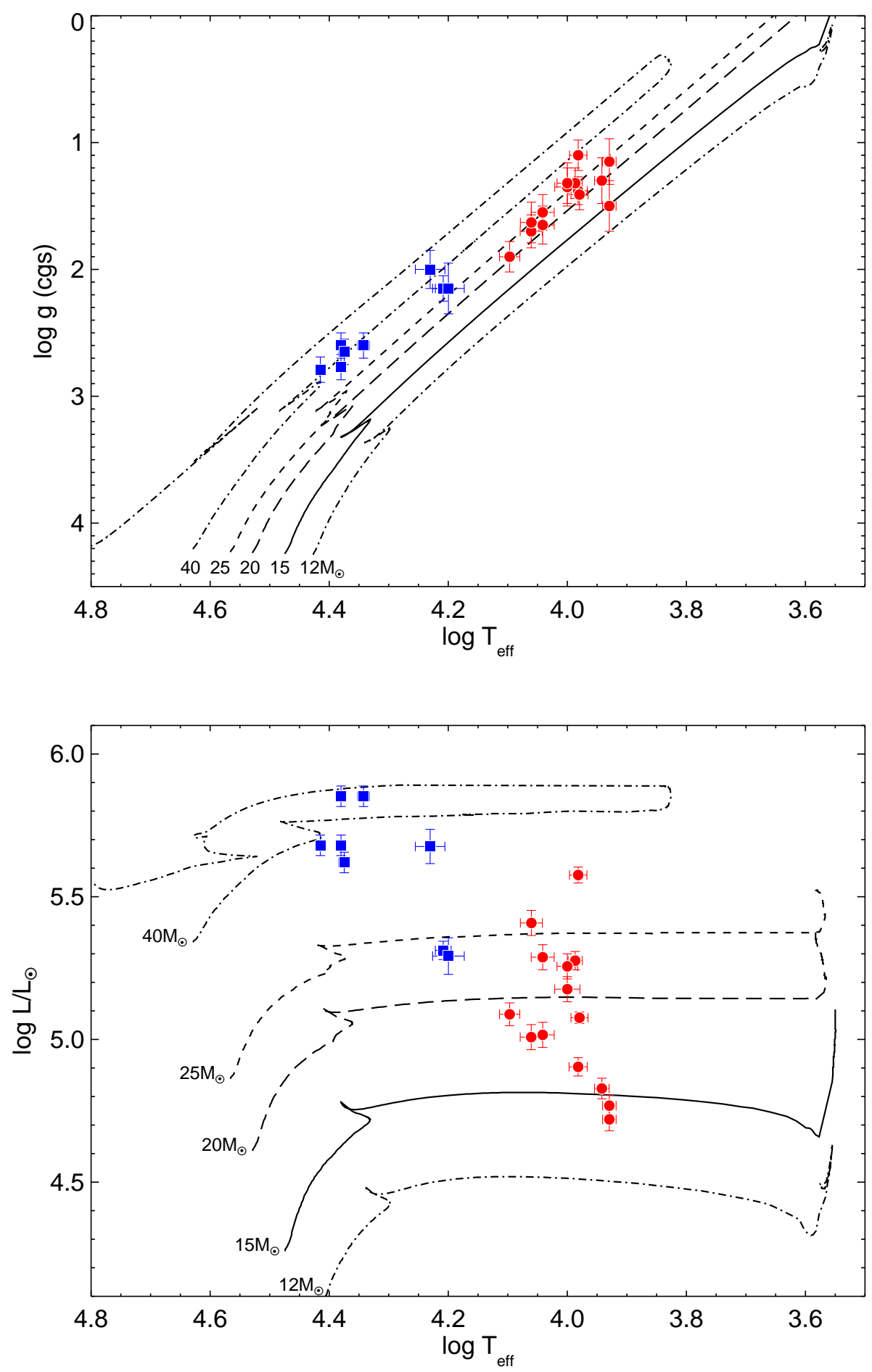

Fig. 10. - The M 33 supergiants of this study in the $\log g-\log T_{\text {eff }}$ plane (top) and in the H-R diagram (bottom). Early B types are shown as solid squares, late B and A types as solid circles. Evolutionary tracks (Meynet \& Maeder 2003) for different ZAMS masses are overplotted. 


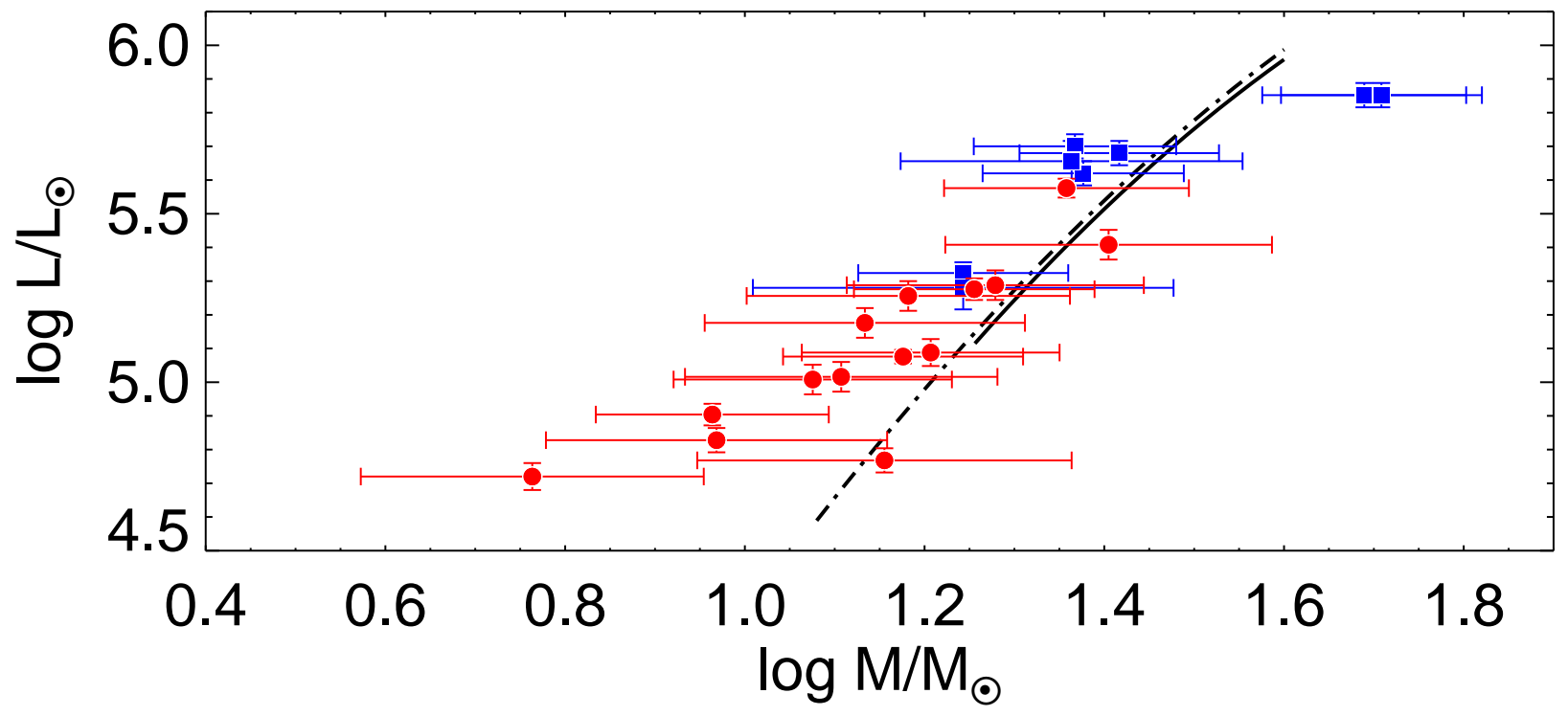

Fig. 11.- The observed mass-luminosity relationship compared with the relationships (dashed: late B and A supergiants, solid: early B supergiants) obtained from the evolutionary tracks of Fig. 11. Spectroscopic masses derived from stellar gravity and radius are used for the M 33 targets of this study. 

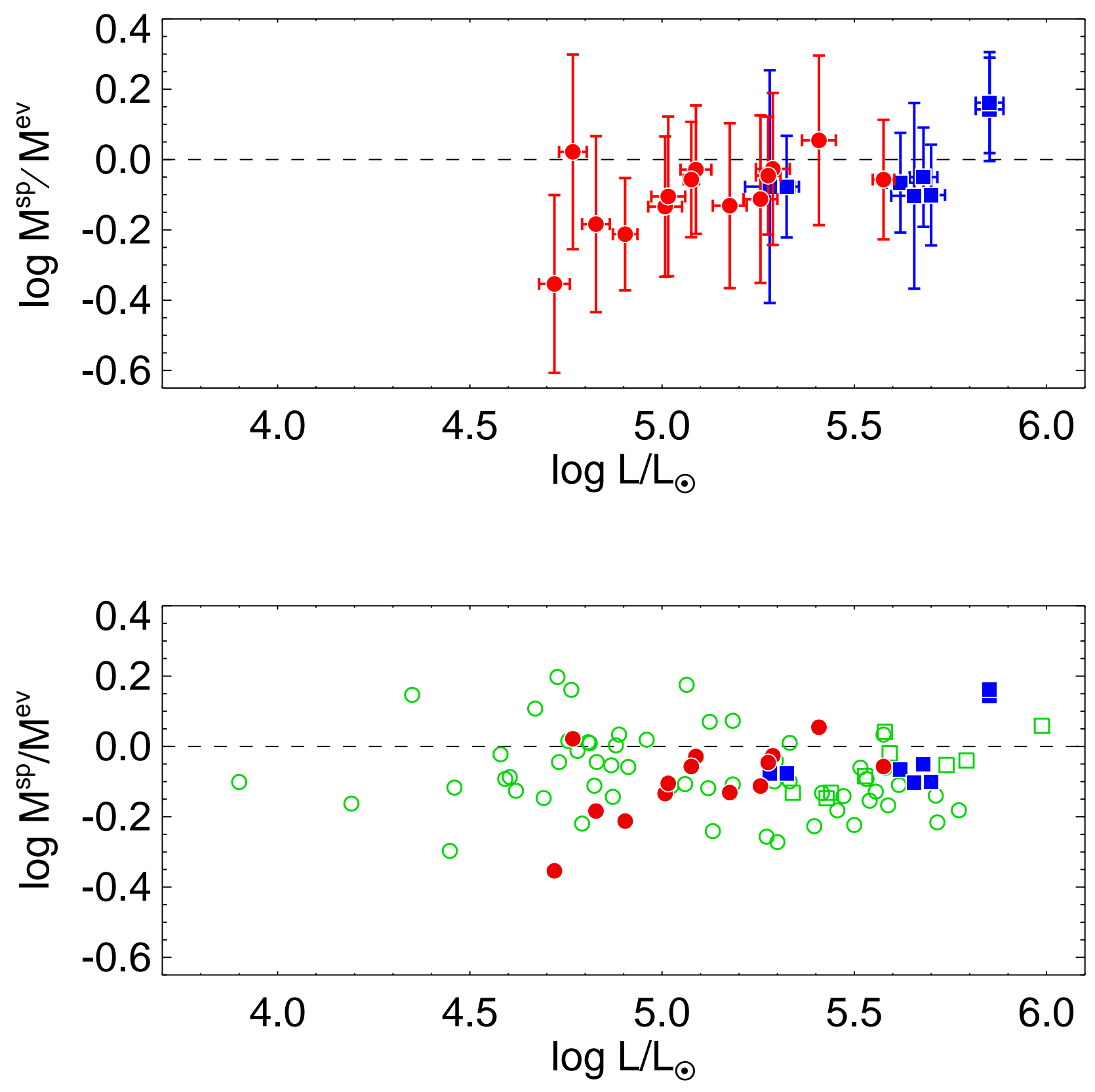

Fig. 12.- Logarithmic ratio of spectroscopic to evolutionary masses as a function of luminosity. The upper diagram contains only the M 33 objects of this study, whereas the lower diagram includes the results by K08 and Urbaneja et al. (2008). 


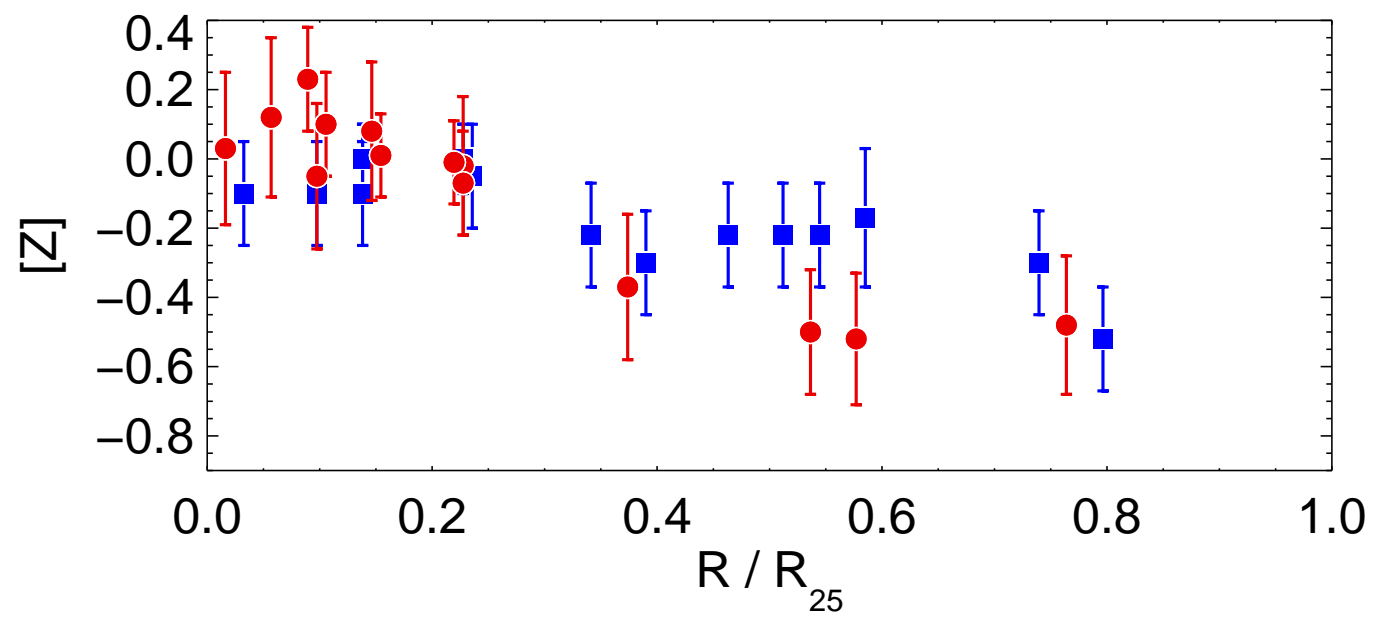

Fig. 13.- Blue supergiant metallicity $[Z]$ as a function of dimensionless angular galactocentric distance. Circles: late B and A supergiants; squares: early B supergiants. 


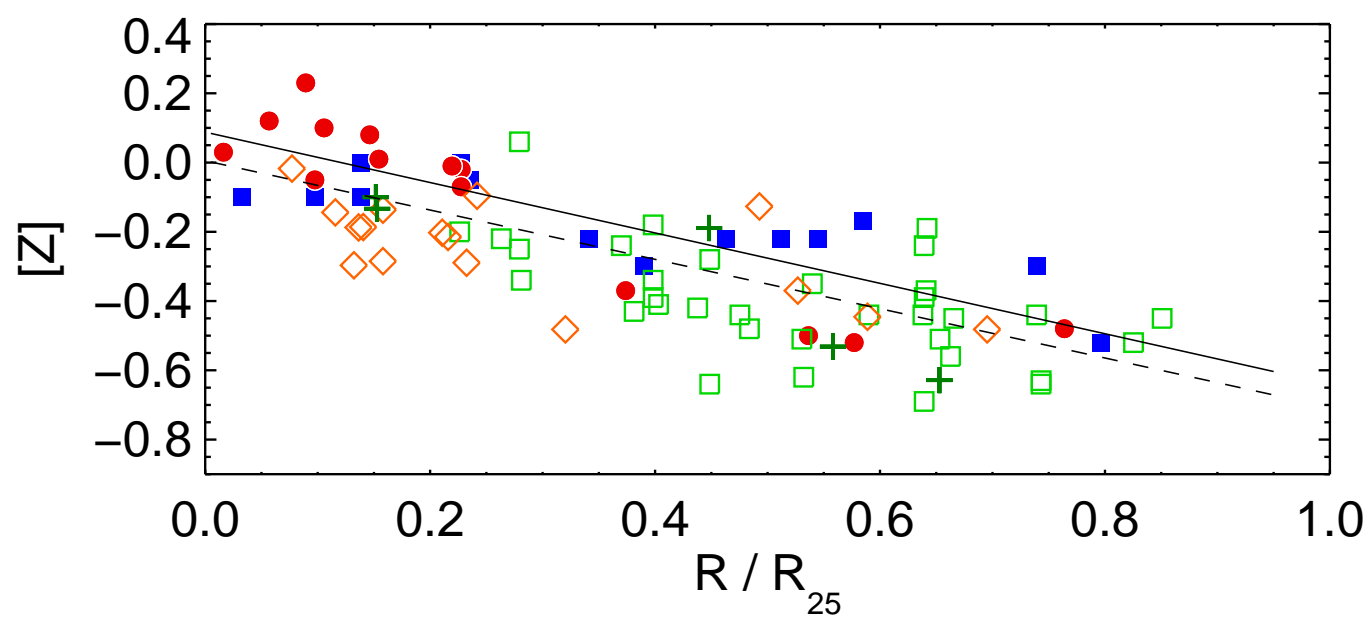

Fig. 14.- Metallicity of blue supergiants, H II regions and Cepheids as a function of dimensionless angular galactocentric distance. A and B supergiants have the same symbols as Fig. 13. Logarithmic oxygen abundances of H II regions in units of the solar value as published by Magrini et al. (2007a) are plotted as open squares. Logarithmic neon abundances of H II regions normalized to the value for B stars in the solar neighbourhood and as obtained from Rubin et al. (2008) are shown as large open diamonds. The metallicity $[Z]$ for beat Cepheids as determined by Beaulieu et al. (2006) are given as crosses. The solid line is the regression for the supergiants only, whereas the dashed lines is the regression for all objects. 


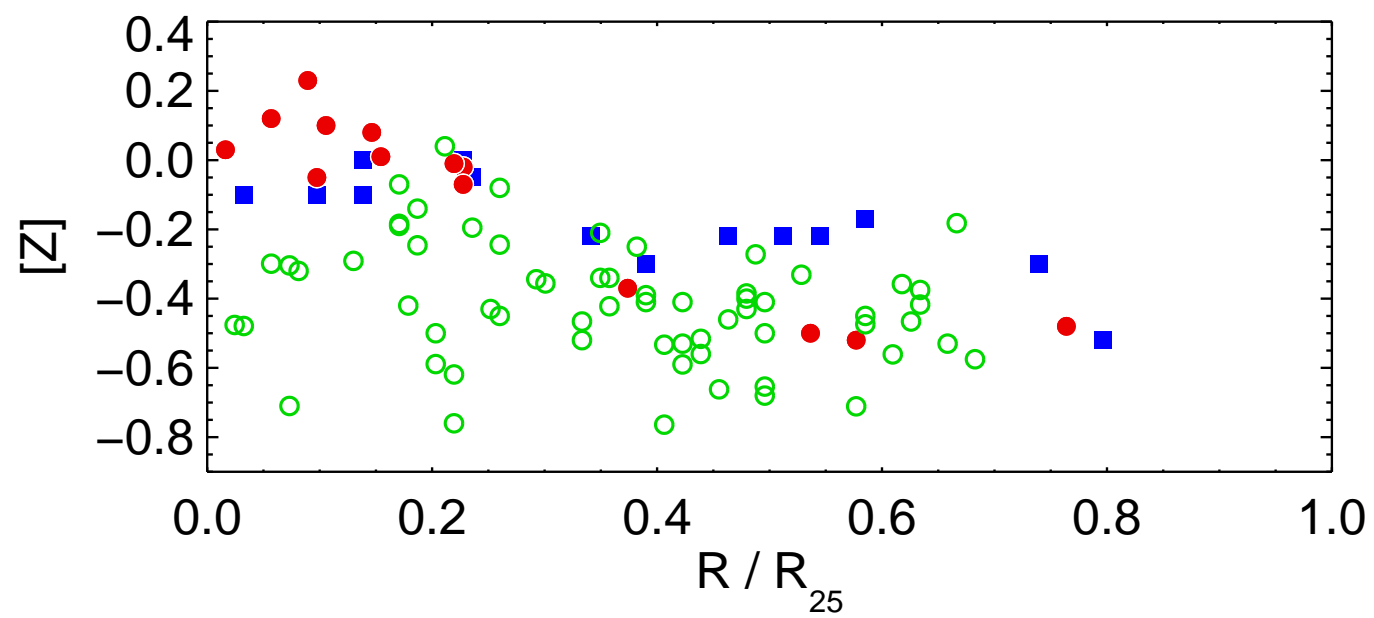

Fig. 15.- Blue supergiant metallicity $[Z]$ as a function of dimensionless angular galactocentric distance compared to the logarithmic oxygen abundances (in units of the solar value) of the H II regions investigated by Rosolowsky \& Simon (2008), open circles. 

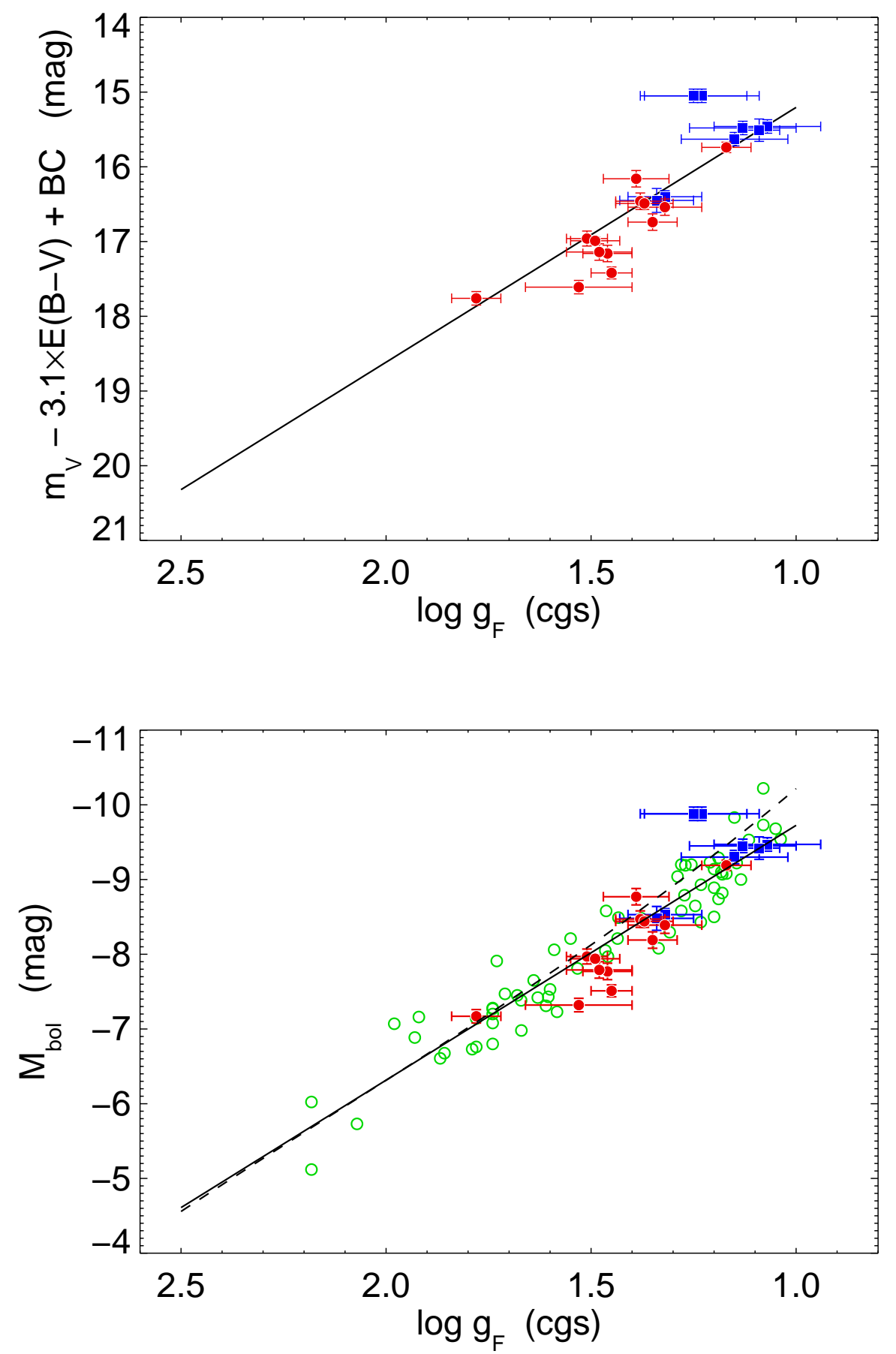

Fig. 16.- FGLR fits of the blue supergiants in M33. Top: Apparent de-reddened bolometric magnitude vs. flux-weighted gravity. Solid circles are late B and A supergiants and solid squares are early B supergiants in M33. The solid line is a linear fit as described in the text. Bottom: Absolute bolometric magnitude vs. flux-weighted gravity. In addition to the M33 targets, objects from nine other galaxies investigated in the studies by K08 and Urbaneja et al. (2008) are also shown. The solid line is the regression FGLR from K08. The dashed curve is the stellar evolution FGLR for Milky Way metallicity. 

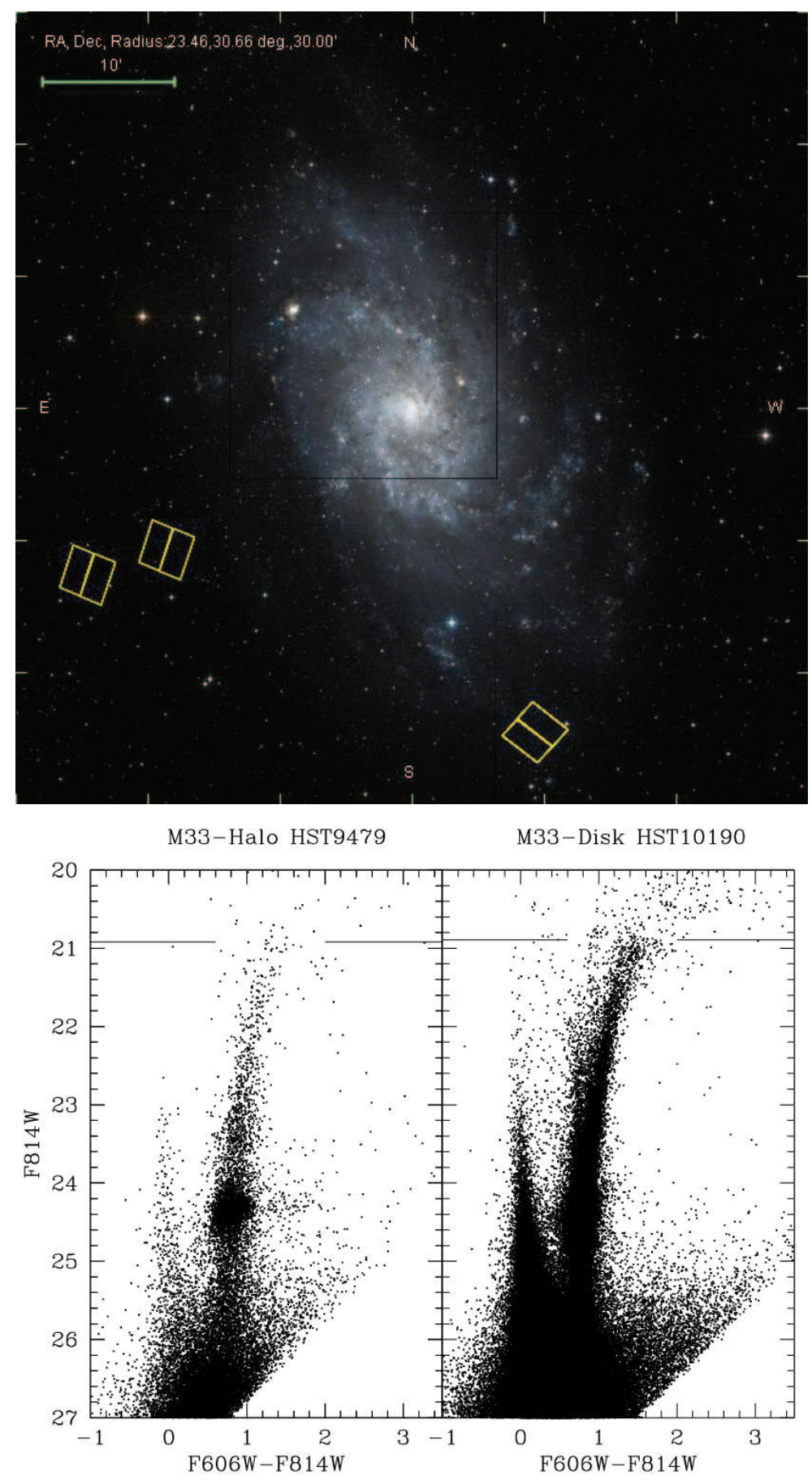

Fig. 17.- Observation of the TRGB in M33. The location of the HST ACS halo fields and the disk field are shown in the top figure. The bottom figure shows the two color-magnitude diagrams obtained from the halo fields and the disk field. The $I$ magnitude of tip of the red giant branch is indicated. 
Table 1. Target Identification

\begin{tabular}{crll}
\hline \hline No. & Massey et al. (2006) ID & \multicolumn{1}{c}{ Alt. ID } & Reference \\
\hline 1 & J013351.20+303224.5 & & \\
2 & J013337.09+303521.6 & & \\
3 & J013340.47+303503.3 & & \\
4 & J013340.84+303822.5 & & \\
5 & J013341.36+303629.6 & \\
6 & J013344.27+304247.2 & & \\
7 & J013344.43+303843.9 & \\
8 & J013344.81+303217.8 & & \\
9 & J013340.55+303158.7 & & \\
10 & J013343.26+303153.8 & & \\
11 & J013340.30+302144.1 & 0755 & \\
12 & J013344.66+303631.6 & B215a & \\
13 & J013229.61+303513.3 & & \\
14 & J013315.62+302949.3 & & $2,3,4$ \\
15 & J013351.56+304005.2 & & \\
16 & J013300.23+302323.7 & 117 A & 2,4 \\
17 & J013339.08+302010.7 & & 4 \\
18 & J013300.86+303504.9 & B38, OB 21-108, UIT0்30 & 2 \\
19 & J013342.06+302142.3 & B287, OB 112-41 \\
20 & J013335.76+310046.9 & B157, UIT 136 & 4 \\
21 & J013333.72+304719.9 & UIT 122 & \\
22 & J013327.35+310056.4 & UIT 103 & \\
\hline & & & \\
\hline
\end{tabular}

Note.

Humphrevs \& Sandage (1980),

Massey et al. (1996)
(1) Ivanov et al. (1993),

(2)

(3) Massey et al. (1995),

(4) 
Table 2. Spectra, Spectral Types, and Spectroscopic Parameters

\begin{tabular}{|c|c|c|c|c|c|c|c|c|c|c|}
\hline No. & $\begin{array}{c}\text { Sp. } \\
\text { Type }\end{array}$ & $\mathrm{S}^{1}$ & $\begin{array}{c}v \sin i \\
\left(\mathrm{~km} \mathrm{~s}^{-1}\right)\end{array}$ & $\begin{array}{c}\zeta^{2} \\
\left(\mathrm{~km} \mathrm{~s}^{-1}\right)\end{array}$ & $R / R_{25}{ }^{3}$ & $\mathrm{~T}^{4}$ & $\begin{array}{l}T_{\text {eff }} \\
(\mathrm{K})\end{array}$ & $\begin{array}{l}\log g \\
(\operatorname{dex})\end{array}$ & $\begin{array}{c}{[Z]} \\
(\operatorname{dex})\end{array}$ & $\begin{array}{c}\log g_{F} \\
(\operatorname{dex})\end{array}$ \\
\hline 1 & B9 & $\mathrm{D}$ & 40 & & 0.23 & $\mathrm{~b}$ & $11000 \pm 500$ & $1.55 \pm 0.14$ & $-0.07 \pm 0.15$ & $1.38 \pm 0.06$ \\
\hline 2 & $\mathrm{~A} 2$ & $\mathrm{D}$ & 27 & & 0.15 & $\mathrm{a}$ & $8500 \pm 225$ & $1.15 \pm 0.15$ & $0.01 \pm 0.12$ & $1.43 \pm 0.10$ \\
\hline 3 & B9 & $\mathrm{D}$ & 53 & & 0.14 & $\mathrm{~b}$ & $11500 \pm 500$ & $1.70 \pm 0.13$ & $0.08 \pm 0.20$ & $1.46 \pm 0.06$ \\
\hline 4 & $\mathrm{~A} 2$ & $\mathrm{D}$ & 25 & & 0.09 & $\mathrm{a}$ & $8500 \pm 225$ & $1.50 \pm 0.20$ & $0.23 \pm 0.15$ & $1.78 \pm 0.06$ \\
\hline 5 & $\mathrm{~A} 2$ & $\mathrm{D}$ & 22 & & 0.11 & $\mathrm{a}$ & $8750 \pm 250$ & $1.30 \pm 0.18$ & $0.10 \pm 0.15$ & $1.53 \pm 0.13$ \\
\hline 6 & B3 & $\mathrm{D}$ & 45 & & 0.14 & $\mathrm{c}$ & $16000 \pm 1000$ & $2.15 \pm 0.20$ & $0.00 \pm 0.10$ & $1.33 \pm 0.09$ \\
\hline 7 & B9 & $\mathrm{D}$ & 30 & & 0.06 & $\mathrm{~b}$ & $11000 \pm 500$ & $1.65 \pm 0.15$ & $0.12 \pm 0.23$ & $1.48 \pm 0.08$ \\
\hline 8 & B8 & $\mathrm{D}$ & 56 & & 0.22 & $\mathrm{~b}$ & $12500 \pm 500$ & $1.90 \pm 0.12$ & $-0.01 \pm 0.12$ & $1.51 \pm 0.05$ \\
\hline 9 & B3 & $\mathrm{D}$ & 58 & & 0.23 & $\mathrm{c}$ & $16000 \pm 1000$ & $2.15 \pm 0.10$ & $0.00 \pm 0.10$ & $1.33 \pm 0.09$ \\
\hline 10 & $\mathrm{~A} 0$ & $\mathrm{D}$ & 42 & & 0.23 & $a, b$ & $10000 \pm 500$ & $1.35 \pm 0.15$ & $-0.02 \pm 0.20$ & $1.35 \pm 0.06$ \\
\hline 11 & A0 & $\mathrm{E}$ & 35 & 30 & 0.54 & $a, b$ & $9600 \pm 340$ & $1.38 \pm 0.11$ & $-0.50 \pm 0.18$ & $1.45 \pm 0.05$ \\
\hline 12 & $\mathrm{~A} 0$ & $\mathrm{E}$ & 50 & 30 & 0.09 & $a, b$ & $9700 \pm 270$ & $1.32 \pm 0.12$ & $-0.05 \pm 0.21$ & $1.37 \pm 0.07$ \\
\hline 13 & $\mathrm{~A} 0$ & $\mathrm{E}$ & 42 & 20 & 0.76 & $a, b$ & $9550 \pm 320$ & $1.41 \pm 0.12$ & $-0.48 \pm 0.19$ & $1.49 \pm 0.06$ \\
\hline 14 & B9 & $\mathrm{E}$ & 35 & 25 & 0.37 & $a, b$ & $10000 \pm 410$ & $1.32 \pm 0.16$ & $-0.37 \pm 0.21$ & $1.32 \pm 0.09$ \\
\hline 15 & B9 & $\mathrm{E}$ & 45 & 30 & 0.01 & $a, b$ & $11500 \pm 500$ & $1.63 \pm 0.16$ & $0.03 \pm 0.22$ & $1.39 \pm 0.08$ \\
\hline 16 & $\mathrm{~A} 0$ & $\mathrm{E}$ & 45 & 30 & 0.57 & $a, b$ & $9600 \pm 328$ & $1.10 \pm 0.12$ & $-0.52 \pm 0.19$ & $1.17 \pm 0.06$ \\
\hline 17 & B3 & $\mathrm{E}$ & 50 & 40 & 0.59 & $\mathrm{c}$ & $17000 \pm 1000$ & $2.00 \pm 0.15$ & $-0.17 \pm 0.20$ & $1.08 \pm 0.05$ \\
\hline $18^{5}$ & B1 & $\mathrm{I}$ & 60 & & 0.46 & $\mathrm{c}$ & $22000 \pm 500$ & $2.60 \pm 0.10$ & $-0.22 \pm 0.15$ & $1.23 \pm 0.14$ \\
\hline $19^{5}$ & B0 & $\mathrm{E}$ & 65 & & 0.54 & $\mathrm{c}$ & $26000 \pm 500$ & $2.79 \pm 0.10$ & $0.00 \pm 0.15$ & $1.13 \pm 0.13$ \\
\hline $20^{5}$ & B0.5 & $\mathrm{E}$ & 70 & & 0.74 & $\mathrm{c}$ & $24000 \pm 500$ & $2.60 \pm 0.10$ & $-0.30 \pm 0.15$ & $1.08 \pm 0.13$ \\
\hline $21^{5}$ & B0.5 & $\mathrm{E}$ & 70 & & 0.35 & $\mathrm{c}$ & $24000 \pm 500$ & $2.77 \pm 0.10$ & $-0.22 \pm 0.15$ & $1.25 \pm 0.13$ \\
\hline $22^{5}$ & B0.7 & $\mathrm{E}$ & 50 & & 0.79 & $\mathrm{c}$ & $23700 \pm 500$ & $2.65 \pm 0.10$ & $-0.52 \pm 0.15$ & $1.15 \pm 0.13$ \\
\hline
\end{tabular}

${ }^{1}$ Spectrograph: D - DEIMOS, E - ESI, I - ISIS

${ }^{2}$ Macroturbulence velocity

${ }^{3} R_{25}=35.40^{\prime}$, the radius of M33 in the plane of the galaxy; this has been corrected for a position angle of $22^{\circ}$ and an inclination angle of $54^{\circ}$



${ }^{5}$ Parameters adopted from Urbaneja et al. (2005b), with the galactocentric distances corrected for the normalization radius and the distance to the galaxy used in this paper 
Table 3. Photometric Properties and Bolometric Corrections

\begin{tabular}{|c|c|c|c|c|c|c|}
\hline $\mathrm{N}$ & $\begin{array}{c}m_{V}^{1} \\
(\mathrm{mag})\end{array}$ & $\begin{array}{l}B-V^{1} \\
(\mathrm{mag})\end{array}$ & $\begin{array}{c}(B-V)_{0} \\
(\mathrm{mag})\end{array}$ & $\begin{array}{c}E(B-V) \\
(\mathrm{mag})\end{array}$ & $\begin{array}{l}M_{V}^{2} \\
(\mathrm{mag})\end{array}$ & $\begin{array}{c}B C \\
(\mathrm{mag})\end{array}$ \\
\hline 1 & 4 & & & & 35 & -0 \\
\hline 2 & & 4 & & & 99 & \\
\hline 3 & 4 & 4 & 20 & 20 & 22 & \\
\hline 4 & 18 & 4 & & & -7 . & \\
\hline 5 & & & 28 & & -7. & 32 \\
\hline 6 & & & & & -7 & \\
\hline 7 & 3 & 0.0 & -0 & 0 & -7 & 85 \\
\hline 8 & & & & & -7 & \\
\hline 9 & 4 & - & -0 . & 0 . & -7.1 & -1 . \\
\hline 10 & 4 & 0 . & -0 . & 0 . & -7.8 & -0 . \\
\hline 11 & 04 & 0.0 & -0 & 0.0 & -7.2 & -0 \\
\hline 12 & 16 & 0.0 & -0.0 & 0.0 & -8.19 & -0. \\
\hline 13 & 04 & 0.1 & -0.0 & 0.13 & -7.71 & -0.2 \\
\hline 14 & $17.120 \pm$ & 0.06 & -0.0 & 0.080 & -8.05 & -0. \\
\hline 15 & $17.102 \pm 0.003$ & 0.039 & $-0.0 \varepsilon$ & $0.120=$ & -8.200 & .060 \\
\hline 16 & $16.440 \pm 0.004$ & $0.142 \pm 0.004$ & $-0.01^{\circ}$ & $0.130 \pm 0.030$ & -8.893 & .020 \\
\hline 17 & $17.209 \pm 0.005$ & -0.07 & -0.15 & $0.075 \pm 0.020$ & -7.95 & -1 \\
\hline 18 & 17.3 & - & -0 & $0.052=$ & -7.7 & -2 \\
\hline 19 & $17.989 \pm 0.0$ & -0.17 & $=0.010$ & $0.012 \pm 0.011$ & $-6.975 \pm 0.034$ & -2.470 \\
\hline 20 & $17.898 \pm 0.005$ & -0.144 & $-0.170 \pm 0.010$ & $0.026 \pm 0.011$ & $-7.113 \pm 0.03$ & -2.34 \\
\hline 21 & $17.754 \pm 0.004$ & $-0.063 \pm 0.004$ & $-0.180 \pm 0.005$ & $0.117 \pm 0.006$ & $-7.539 \pm 0.019$ & $-2.340 \pm 0.084$ \\
\hline 22 & $18.035 \pm 0.005$ & $-0.154 \pm 0.005$ & $-0.180 \pm 0.005$ & $0.026 \pm 0.007$ & $-6.976 \pm 0.022$ & $-2.320 \pm 0.084$ \\
\hline
\end{tabular}

${ }^{1}$ Adopted from Massey et al. (2006)

${ }^{2}$ Computed with distance modulus of $24.93 \pm 0.11$ mag (this work) 
Table 4. Bolometric Magnitudes, Luminosities, and Stellar Masses

\begin{tabular}{rccrrr}
\hline \hline No. & $\begin{array}{c}M_{\text {bol }} 1 \\
(\mathrm{mag})\end{array}$ & $\begin{array}{c}\log \left(L / L_{\odot}\right) \\
(\mathrm{dex})\end{array}$ & $\begin{array}{c}R \\
\left(R_{\odot}\right)\end{array}$ & $\begin{array}{r}M_{\text {spec }} \\
\left(M_{\odot}\right)\end{array}$ & $\begin{array}{c}M_{\text {evol }} \\
\left(M_{\odot}\right)\end{array}$ \\
\hline 1 & $-8.51 \pm 0.11$ & $5.30 \pm 0.04$ & $121 \pm 13$ & $19.0 \pm 8.8$ & $20.2 \pm 0.7$ \\
2 & $-7.05 \pm 0.10$ & $4.73 \pm 0.04$ & $106 \pm 8$ & $5.8 \pm 3.2$ & $13.1 \pm 0.4$ \\
3 & $-7.77 \pm 0.11$ & $5.01 \pm 0.04$ & $81 \pm 8$ & $11.9 \pm 5.1$ & $16.2 \pm 0.5$ \\
4 & $-7.17 \pm 0.09$ & $4.77 \pm 0.04$ & $112 \pm 8$ & $14.3 \pm 8.8$ & $13.6 \pm 0.3$ \\
5 & $-7.32 \pm 0.09$ & $4.83 \pm 0.04$ & $113 \pm 8$ & $9.3 \pm 5.1$ & $14.2 \pm 0.4$ \\
6 & $-8.50 \pm 0.16$ & $5.30 \pm 0.06$ & $58 \pm 9$ & $17.5 \pm 12.5$ & $20.9 \pm 1.0$ \\
7 & $-7.79 \pm 0.11$ & $5.02 \pm 0.04$ & $89 \pm 10$ & $12.8 \pm 6.3$ & $16.3 \pm 0.5$ \\
8 & $-7.97 \pm 0.11$ & $5.09 \pm 0.04$ & $75 \pm 7$ & $16.1 \pm 6.3$ & $17.2 \pm 0.5$ \\
9 & $-8.54 \pm 0.08$ & $5.32 \pm 0.03$ & $58 \pm 4$ & $17.5 \pm 5.4$ & $20.9 \pm 0.5$ \\
10 & $-8.19 \pm 0.11$ & $5.18 \pm 0.04$ & $129 \pm 15$ & $13.6 \pm 6.9$ & $18.4 \pm 0.6$ \\
11 & $-7.51 \pm 0.08$ & $4.90 \pm 0.03$ & $103 \pm 9$ & $9.2 \pm 3.2$ & $15.0 \pm 0.3$ \\
12 & $-8.44 \pm 0.08$ & $5.28 \pm 0.03$ & $154 \pm 11$ & $18.0 \pm 6.5$ & $20.0 \pm 0.5$ \\
13 & $-7.94 \pm 0.05$ & $5.08 \pm 0.02$ & $127 \pm 9$ & $15.0 \pm 5.4$ & $17.1 \pm 0.3$ \\
14 & $-8.39 \pm 0.11$ & $5.26 \pm 0.04$ & $142 \pm 14$ & $15.2 \pm 7.8$ & $19.7 \pm 0.7$ \\
15 & $-8.77 \pm 0.11$ & $5.41 \pm 0.04$ & $128 \pm 13$ & $25.4 \pm 13.2$ & $22.4 \pm 0.8$ \\
16 & $-9.19 \pm 0.07$ & $5.58 \pm 0.03$ & $223 \pm 17$ & $22.8 \pm 8.4$ & $26.0 \pm 0.6$ \\
17 & $-9.44 \pm 0.15$ & $5.68 \pm 0.06$ & $80 \pm 12$ & $23.1 \pm 12.7$ & $29.3 \pm 1.7$ \\
18 & $-9.88 \pm 0.09$ & $5.85 \pm 0.04$ & $58 \pm 4$ & $48.9 \pm 14.6$ & $35.2 \pm 1.4$ \\
19 & $-9.45 \pm 0.09$ & $5.68 \pm 0.04$ & $34 \pm 2$ & $26.1 \pm 7.6$ & $29.3 \pm 1.0$ \\
20 & $-9.45 \pm 0.09$ & $5.68 \pm 0.04$ & $40 \pm 2$ & $23.3 \pm 6.9$ & $29.4 \pm 1.0$ \\
21 & $-9.88 \pm 0.09$ & $5.85 \pm 0.04$ & $49 \pm 3$ & $51.1 \pm 15.0$ & $35.2 \pm 1.3$ \\
22 & $-9.30 \pm 0.09$ & $5.62 \pm 0.04$ & $38 \pm 2$ & $23.8 \pm 7.0$ & $27.7 \pm 0.9$ \\
\hline & & & & &
\end{tabular}

${ }^{1}$ Computed with distance modulus of $24.93 \pm 0.11 \mathrm{mag}$ (this work) 